\title{
Perturbation-based prediction of vibration phase shift along fluid-conveying pipes due to Coriolis forces, nonuniformity, and nonlinearity
}

Thomsen, Jon Juel; Fuglede, Niels

Published in:

Nonlinear Dynamics

Link to article, DOI:

$10.1007 / \mathrm{s} 11071-019-04934-6$

Publication date:

2020

Document Version

Peer reviewed version

Link back to DTU Orbit

Citation (APA):

Thomsen, J. J., \& Fuglede, N. (2020). Perturbation-based prediction of vibration phase shift along fluidconveying pipes due to Coriolis forces, nonuniformity, and nonlinearity. Nonlinear Dynamics, 99, 173-199. https://doi.org/10.1007/s11071-019-04934-6

\section{General rights}

Copyright and moral rights for the publications made accessible in the public portal are retained by the authors and/or other copyright owners and it is a condition of accessing publications that users recognise and abide by the legal requirements associated with these rights.

- Users may download and print one copy of any publication from the public portal for the purpose of private study or research.

- You may not further distribute the material or use it for any profit-making activity or commercial gain

- You may freely distribute the URL identifying the publication in the public portal 


\title{
Perturbation-based prediction of vibration phase shift along fluid-conveying pipes due to Coriolis forces, nonuniformity, and nonlinearity
}

\author{
Jon Juel Thomsen* and Niels Fuglede \\ Department of Mechanical Engineering, Technical University of Denmark, DK-2800 Kgs. Lyngby, Denmark \\ *Email: jjt@mek.dtu.dk; ORCID ID: 0000-0003-2311-7888
}

\begin{abstract}
Flexural vibrations of a fluid-conveying pipe are investigated theoretically, with special consideration to the spatial shift in vibration phase caused by fluid flow and various imperfections. The latter includes small nonuniformity or asymmetry in stiffness, mass, or damping, and weak stiffness and damping nonlinearity. Besides contributing general understanding of wave propagation in elastic media with gyroscopic forces, this is relevant for the design, control, and trouble-shooting of phase-shift measuring devices like Coriolis mass flowmeters. A multiple time scaling perturbation analysis is employed with a simple model of a fluid-conveying pipe with relevant imperfections, resulting in simple analytical expressions for the prediction of phase shift. For applications like Coriolis flowmetering this allows for readily examining effects of a variety of relevant features, like small sensors and actuators, production inaccuracies, mounting conditions, wear, contamination, and corrosion. To second order of accuracy, only mass flow and asymmetrically distributed damping are predicted to introduce spatial phase shift, while nonuniformly distributed linear mass and stiffness, symmetrically distributed linear damping, and uniformly nonlinear stiffness and damping are all negligible in comparison. The analytical predictions are illustrated by examples, and validated with excellent agreement against numerical analysis for realistic magnitudes of parameters.
\end{abstract}

Keywords mechanical nonlinearity, perturbation analysis, fluid-conveying pipes, spatial phase shift, structural imperfection, Coriolis flowmeter

Acknowledgments The authors are thankful to Siemens Flow Instruments in Denmark for stimulating problems and discussions, technical assistance with experiments, and partial financial support.

\section{Introduction}

The phase shift in oscillation between two points of a vibrating elastic beam or pipe is important for some applications. For a linear beam with mass- or stiffness-proportional damping standing waves can exist, with all points vibrating in either phase or antiphase. However, several factors can introduce nontrivial spatial phase dependencies, and thus non-standing, travelling waves. Non-proportional damping is one such factor. Fluid flow another, as utilized in Coriolis flowmeters, where the phase change between two points at a vibrated pipe is measured and related to mass flow through the pipe. Ideally, for such a flowmeter, the phase shift depends only on mass flow. But many other factors could potentially be a source of phase shift, and thus mistaken for mass flow. This work seeks to clarify how typical linear and nonlinear mechanical effects can possibly lead to phase shifts for a vibrating pipe with fluid flow.

In [1] a systematic perturbation approach was presented for deriving analytical expressions that relate phase shift to parameters characterizing vibrating pipes conveying fluid flow and possible small imperfections. Here we simplify the procedure, and include more types of imperfections, in particular axially nonuniform distribution of mass, stiffness, and damping, and nonlinear stiffness and damping. The result is a simple approximate analytical prediction (Eq. (52)), accurate to second order in the small parameter characterizing imperfection magnitude, for calculating how phase shift depends on the imperfections considered. The expression is validated against numerical simulation for some relevant and illustrative cases, and against some existing reported experimental results.

Aspects of vibrations and stability of fluid-conveying pipes have been investigated for a long time [2-8], apparently with the first reported derivations of the correct equations of motion and 
analysis reported by F.-J. Bourriêres in 1939 [4]. Some of these works were motivated by Coriolis flowmetering applications, providing valuable insights into how mass flow and also certain imperfections influence phase shift, see e.g. the overviews [9-11]. Several studies derive analytical or semi-analytical predictions for phase shifts in the context relevant here. Typically the analytical expressions are for the ideal case [12], or require numerical solution of an eigenvalue problem $[13,14]$, or the results are not validated against numerical simulation or experimentally. Raszillier and Durst [12] used a perturbation-like approach to derive analytical expressions for the phase shift for fluid-conveying pipes, but did not consider imperfections other than mass flow. Effects of imperfect supports on phase shift was considered in [1], using perturbation analysis in a manner similar to what is used in the present work. Effects of mechanical vibrations on measurement accuracy has been considered [15], as has effects of pulsating flow speed [16-19], sensor and actuator mass [10], temperature [10,20], and multi-phase flow [21]. Kutin \& Bajsic [22,23] employed Taylor-expanded (in fluid velocity) Galerkinsolutions to calculate analytical predictions for Coriolis flowmeter stability boundaries, and for phase shifts in the ideal and some non-ideal cases (nonlinear flow, axial force, and added mass).

The effect of structural nonlinearity on phase shift for pipes with fluid flow seems not to have received much attention in the literature. This may be partly due the theoretical difficulties that comes with nonlinearity, and partly due to unawareness of the potential practical importance. One might think the very small displacement amplitudes typical in Coriolis flowmetering would imply that nonlinearity can safely be ignored. However, some of the strongest sources of nonlinearity in real structures can become significant even at very small displacement amplitude. This concerns e.g. the stiffness nonlinearity associated with midplane stretching (relevant for pipes with fixed ends) or mechanical clearance (pipe rattling against rigid obstacle), and also with certain types of nonlinear damping (e.g. dry friction at pipe clamps or connections). Also, it is well-known that even weak nonlinearity can have a strong effect on vibration response, to an extent where linear theory does not even qualitatively capture the response correctly, as e.g. with nonlinear modal interaction [24-26], when one vibration mode is resonantly excited (as with Coriolis flowmetering), and the natural frequency of this mode is related by a specific ratio of small integers to the natural frequency of another mode. Thus consideration of nonlinearity is relevant.

Stiffness nonlinearity from midplane stretching was considered with Coriolis flowmetering in [27], however without considering the effect on phase shift (but only the additional frequency components). The same study also considers nonlinear damping, though only in an unphysical form proportional to squared velocity (i.e. without multiplying with the sign of the velocity like with physically meaningful "quadratic damping"), and without considering phase shift effects. In the present work we consider the effect on phase shift of quadratic-cubic nonlinear stiffness (generically representative as the dominating nonlinear terms for most smooth stiffness nonlinearities), and general velocity-dependent nonlinear damping.

Recently [28] investigated several aspects of nonlinear vibrations and stability for a mathematical model of a curved micro Coriolis flowmeter, taking into account geometrical nonlinearity in form of cubic stiffness terms. Using Galerkin discretization in terms of mode shapes for fixed boundaries, the spatial phase shift was calculated numerically for a specific example, along with system natural frequencies and flow stability thresholds. For the numerical example a linear dependency between phase shift and mass flow was observed, even in the presence of nonlinear stiffness. The present work puts more general theoretical support to this specific observation.

Below we present and exemplify a systematic approach for calculating simple approximate analytical expression for spatial phase shifts of pipe vibrations, caused by fluid flow and various small linear and nonlinear imperfections, using a greatly simplified generic model of real 
flowmeters. The mathematical model for transverse vibrations of the pipe takes the form of a nonlinear partial integro-differential equation of motion, with non-constant coefficients and external time-harmonic excitation. Spatial discretization into a finite set of approximating ordinary nonlinear differential equations is obtained using Galerkin expansion in terms of "perfect-pipe" mode shapes. The resulting set of ordinary nonlinear differential equations is then solved using multiple scales perturbation analysis, considering all imperfections as small perturbations to the perfect pipe. For the main case of practical interest in Coriolis flowmetering, i.e. sharply resonant excitation of the lowest mode, using just the two lowest vibration modes proves sufficient for reliably predicting frequency response as well as phase shift for the pipe: The lowest mode is directly excited, and thus strongly present, while the second mode is indirectly and much more weakly excited by weak Coriolis forces from the flowing fluid and possibly other imperfections; As is confirmed by numerical simulation, all higher modes affect the response at a level several orders of magnitude lower.

The modeling and solution procedure employed is similar to what was used in $[1,29]$, except that in the present work more and other kinds of imperfections are considered, and that the analysis is further simplified as compared to [1]: The perturbation analysis was there applied directly to the partial differential equation of motion, whereafter Galerkin expansion is employed, while in the present work Galerkin expansion is employed first, as in also [10].

The simple model and approximate analysis is targeted towards the study of general effects, which may carry over to real flowmeters, not towards specific flowmeter design. For example, the conclusion, for the simple model, that "only the spatially asymmetric part of distributed damping contributes to measured phase shift" may well carry over to real flowmeters of complicated geometry; at least the simple model allows such a hypothesis to be suggested, which is testable experimentally or with detailed numerical simulation for specific, real flowmeters. All of the simplifying assumptions made in this study hold approximately for applications such as Coriolis flowmetering under typical operating conditions. Many real industrial flowmeters have two curved measurement pipes, and so the straight single-pipe model employed here may appear somewhat academic. This is by intention, since the simplified geometry allows for simple, transparent analytical expressions for the quantities of primary interest. These allow for deducing conclusions on various effects of interest, which can then be used for posing hypotheses for real flowmeters, to be tested experimentally or by detailed numerical simulation of coupled fluid-structure interaction models [17,30-35].

Section 2 defines the system and the "perfect" and "imperfect" (i.e. nonuniform and nonlinear) pipe, and sets up and discusses the fundamental equation of motion. Section 3 derives and interprets the primary resonant response mostly relevant for applications, based on a multiple scales perturbation analysis of an approximating set of equations of motion for the nonlinear and nonuniform pipe. Based on the approximate response calculation, Section 4 derives rather simple analytical expressions for predicting spatial phase shift in pipe vibrations in dependency of the parameters describing pipe imperfection, and discusses implications in terms of sources of measurement errors for Coriolis flowmeter applications. Section 5 validates the simplified analytical predictions of phase shift effects, by comparing against results of numerical simulation of the underlying full (i.e. unapproximated) system for cases corresponding to, respectively nonuniform linear damping, mass, and stiffness, and nonlinear damping and stiffness. Finally Section 6 concludes on how the considered imperfections, besides fluid flow, affect spatial shifts in vibration phase. 


\section{Mathematical model}

\subsection{The system}

Fig. 1(a) shows an infinitesimal element of the elastic beam in Fig. 1(b,c), modeling a single, straight Coriolis flowmeter pipe. At time $\tilde{t}$ and longitudinal coordinate $\tilde{x}$, the transverse and longitudinal deformation of the pipe axis is $\tilde{u}$ and $\tilde{w}$, respectively. The pipe element is affected by reaction forces $\Pi$ from the fluid, and transverse external forces $\tilde{P}(\tilde{x}, \tilde{t})$, e.g. from flowmeter actuators; in particular we consider time-harmonic pipe actuation at $\tilde{x}=\tilde{x}_{p}$ having frequency $\tilde{\Omega}$ and force amplitude $\tilde{p}$. The fluid is assumed to have constant mass $m_{f}$ per unit pipe length, and to (plug-) flow through the pipe at a speed $v$ which is everywhere the same inside the pipe, and changing only negligibly during each pipe vibration cycle (in [18] effects of pulsating flow speed $v=v(t)$ are analyzed similarly as below).

The pipe is either hinged or clamped at both ends, cf. Fig. 1(b,c). The supports can be axially fixed, introducing midplane stretching and thus a nonlinear coupling between axial forces and transverse deformation, as could be significant with a straight or slightly curved flowmeter pipe in a stiff frame. The pipe has undeformed length $l$, with its end pre-tensioned or -compressed axially a distance $\eta l,|\eta| \ll 1$, within axially fixed supports, where $\eta$ is positive for tension and negative for compression. Hinged supports are included for their convenience (due to the simple linear mode shapes for a uniform pipe) in illustrating and interpreting analytical results, whereas clamped supports may be more realistic for applications. The equation of motion is solved for both types of boundary conditions, and other types of boundary conditions could be analyzed similarly ( $[1,36]$ investigate effects of imperfect boundary conditions). Imperfections considered in this study include slight axial variations in pipe mass per unit length $m_{p}(\tilde{x})=m_{p 0}+\Delta m_{p}(\tilde{x})$, in bending stiffness $E I(\tilde{x})=E I_{0}+\Delta E I(\tilde{x})$, and in axial stiffness $E A(\tilde{x})=E A_{0}+\Delta E A(\tilde{x})$, where here subscript zero indicates the constant part of each property (e.g. it's mean value over $\tilde{x} \in[0, l]$, or a value typical for most of the pipe length), and $\Delta$ denotes property variation. The constant or uniform part of the mass per unit fluid-filled pipe is $m_{0}=m_{p 0}+m_{f}$. Also, there can be weak distributed transverse and rotational linear viscous damping with coefficients $\tilde{c}_{u}(\tilde{x})$ and $\tilde{c}_{\theta}(\tilde{x})$, and linear external stiffness (additional to pipe bending stiffness) $\tilde{k}_{u}(\tilde{x})$ and $\tilde{k}_{\theta}(\tilde{x})$. All these functions of $\tilde{x}$ can be discontinuous, allowing for examining effects of e.g. mounted sensors and actuators, production inaccuracies,

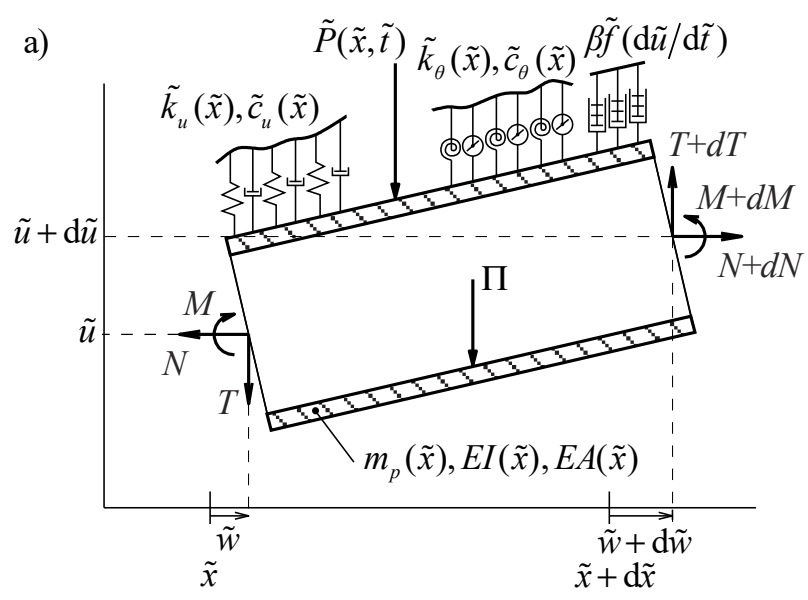

b)

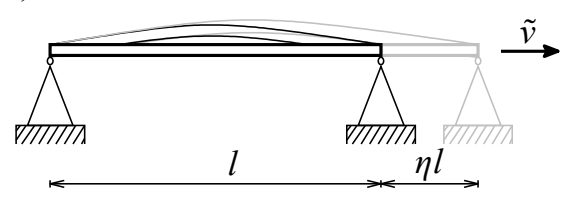

c)

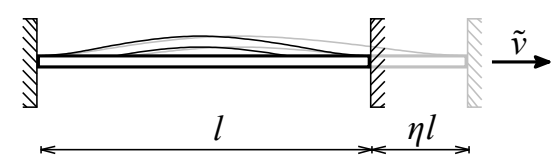

Fig. 1 (a) Infinitesimal pipe element of a (b) hinged or (c) clamped pre-tensioned or -compressed pipe 
mounting conditions, and wear, contamination, and corrosion. The addition of small and uniformly distributed generalized damping $\beta \tilde{f}(\mathrm{~d} \tilde{u} / \mathrm{d} \tilde{t})$ allows for examining effects of nonlinear velocity-dependent dissipation, e.g. quadratic damping; the function $\tilde{f}$ is assumed to be essentially nonlinear (i.e. vanishing along with its first derivative at $\dot{u}=0$ ), and must be continuous in $\dot{u}$ for the relevant range of velocities.

\subsection{Equation of motion}

The equation of motion governing finitely small transverse pipe vibrations $\tilde{u}(\tilde{x}, \tilde{t})$ can be derived using Newton's second law or Hamilton's principle, see e.g. [5,12,37] for details. The result can be written in the following nondimensional form, which is an extension of what can be found in $[1,18,23,37]$ :

$$
\begin{gathered}
\ddot{u}+u^{\prime \prime \prime \prime}+\varepsilon\left[\Delta m(x) \ddot{u}+\left(\Delta e(x) u^{\prime \prime}\right)^{\prime \prime}+\alpha\left(2 v \dot{u}^{\prime}+v^{2} u^{\prime \prime}\right)-\mu^{2}\left(\eta+\frac{1}{2} \int_{0}^{1}\left(u^{\prime}(\xi, t)\right)^{2} d \xi\right) u^{\prime \prime}\right. \\
\left.+\gamma u^{2}+L_{k} u+L_{c} \dot{u}+\beta f(\dot{u})\right]=\varepsilon p \delta\left(x-x_{p}\right) \cos (\Omega t),
\end{gathered}
$$

where $u=u(x, t)$ is the transverse deflection at time $t, x \in[0,1]$ is the axial coordinate, $\delta(x)$ Dirac's delta function, $L_{k}$ and $L_{c}$ are linear spatial differential operators describing additional/external linear stiffness and distributed linear damping, respectively:

$$
L_{k}=k_{u}(x)-\frac{\mathrm{d}}{\mathrm{d} x}\left(k_{\theta}(x) \frac{\mathrm{d}}{\mathrm{d} x}\right), \quad L_{c}=c_{u}(x)-\frac{\mathrm{d}}{\mathrm{d} x}\left(c_{\theta}(x) \frac{\mathrm{d}}{\mathrm{d} x}\right),
$$

the boundary conditions for hinged supports are:

$$
u(0, t)=u(1, t)=u^{\prime \prime}(0, t)=u^{\prime \prime}(1, t)=0,
$$

while for clamped supports:

$$
u(0, t)=u(1, t)=u^{\prime}(0, t)=u^{\prime}(1, t)=0,
$$

and all parameters, variables, and functions are nondimensional:

$$
\begin{gathered}
x=\frac{\tilde{x}}{l}, \quad u=\frac{\tilde{u}}{l}, \quad t=\tilde{\omega}_{0} \tilde{t}, \quad v=\frac{\tilde{v}}{l \tilde{\omega}_{0}}, \quad p=\frac{\tilde{p}}{m_{0} l^{2} \tilde{\omega}_{0}^{2}}, \quad \Omega=\frac{\tilde{\Omega}}{\tilde{\omega}_{0}}, \\
\alpha=\frac{m_{f}}{m_{0}}, \quad \Delta m(x)=\frac{\Delta m_{p}(l x)}{m_{0}}, \quad \Delta e(x)=\frac{\Delta E I(l x)}{E I_{0}}, \\
\mu=\frac{l}{\sqrt{E I_{0} / E A_{m}}}, \quad \gamma=\frac{\tilde{\gamma} l}{m_{0} l \tilde{\omega}_{0}^{2}}, \quad k_{u}(x)=\frac{\tilde{k}_{u}(l x)}{m_{0} \tilde{\omega}_{0}^{2}}, \quad k_{\theta}(x)=\frac{\tilde{k}_{\theta}(l x)}{m_{0} l^{2} \tilde{\omega}_{0}^{2}}, \\
c_{u}(x)=\frac{\tilde{c}_{u}(l x)}{m_{0} \tilde{\omega}_{0}}, \quad c_{\theta}(x)=\frac{\tilde{c}_{\theta}(l x)}{m_{0} l^{2} \tilde{\omega}_{0}}, \quad f(\dot{u})=\frac{\tilde{f}\left(\omega_{0} l \dot{u}\right)}{m_{0} l \tilde{\omega}_{0}^{2}} .
\end{gathered}
$$

In (1)-(5) dots and primes denote differentiation w.r.t. to $t$ and $x$, respectively, $\varepsilon$ is a "bookkeeping" parameter marking terms of smaller order of magnitude, subscripts $p, f, u, \theta$ denote "pipe", "fluid", "transverse", "rotational", respectively, and $1 / E A_{m}=\frac{1}{l} \int_{0}^{l}(E A(\tilde{x}))^{-1} \mathrm{~d} \tilde{x}$ is the mean axial pipe flexibility. Time $t$ is nondimensionalized by the characteristic frequency 
$\tilde{\omega}_{0}=\sqrt{E I_{0} / m_{0} l^{4}}$, the axial coordinate $x$ and transverse deflection $u$ by the pipe length $l$, and flow speed $v$ by the characteristic wave speed $\tilde{\omega}_{0} l$.

Equation (1) is a time-harmonically excited partial differential equation of motion, nonlinear (for nonzero $\mu, \gamma$, or $\beta$ ), and with spatially non-constant coefficients (for non-constant $\Delta m, \Delta e$, $L_{k}$, or $\left.L_{c}\right)$.

\subsection{Physical meaning of nondimensional parameters and terms}

The external pipe actuation is described by the normalized amplitude $p$ and frequency $\Omega$ of a time-harmonic force at $\left.x=x_{p} \in\right] 0,1[$. The function $\Delta e(x)$ describes the normalized nonuniformity in pipe bending stiffness, i.e. the relative deviation along the axis from the constant part of the bending stiffness. Any physically conceivable variation $\Delta e(x)$ can be chosen, even discontinuous (as with abrupt changes in cross section or material, and along with suitable interface conditions of deflection and slope continuity), as long as its magnitude is small compared to unity. Similarly, the function $\Delta m(x)$ describes the nonuniformity in distribution of (fluid-empty) pipe mass, normalized by the constant part $m_{0}$ of the mass of the fluid-filled pipe; the same value is used to normalize the fluid mass $\alpha$ per unit length, so that $\alpha \in[0,1[$, with $\alpha \rightarrow 0$ for a light gas, while $\alpha \rightarrow 1$ for a very heavy fluid or light pipe. A nonuniform pipe can have any variation $\Delta m(x)$, even discontinuous (as with abrupt changes in cross section or material) or singular (as with added point masses), provided $\Delta m$ is small in regular intervals, and integrates over $x$ at any singularity to a small value (e.g. a point mass should be small compared to the total fluid-filled pipe mass).

Nonlinear midplane stretching is expressed by the parameter $\mu>0$. The denominator in the definition of $\mu$ equals the pipe (mean or effective) radius of gyration $r_{0}=\sqrt{I_{0} / A_{m}}$, and thus $\mu=l / r_{0}$ is the pipe's slenderness ratio; the Bernoulli-Euler assumptions employed for (1) holds for "slender pipes", e.g. $\mu \tilde{\lambda} / l>200$ where $\tilde{\lambda}$ is the wavelength of the highest active vibration mode. More slender pipes have larger values of $\mu$, implying that relatively more of the transverse stiffness comes from midplane stretching and pre-tension, and less from bending stiffness.

Linear translational and rotational stiffness and damping is described by the operators $L_{k}$ and $L_{c}$, respectively. They are defined in terms of functions $k_{u}, \theta(x)$ and $c_{u}, \theta(x)$, describing the axial distribution of, respectively, stiffness (additional to the pipe bending stiffness) and viscous damping per unit length. These distributions can be nonuniform and even discontinuous, or possess singularities, but should be small compared to unity in $x$-integrated magnitude. For example, $k_{u}(x)=k_{u 0}+k_{u} \delta\left(x-x_{k u}\right),\left|k_{u 0,1}\right| \ll 1$, models a particular distributed transverse stiffness, the first term describing the uniform part, and the second a transverse spring localized $x=x_{k u}$. The first two terms in (1) represent, respectively, the uniform transverse inertia of the pipe and fluid, and the uniform bending stiffness of the pipe. All remaining terms are small, as indicated by the factor $\varepsilon$. The first and second term within the bracket represents, respectively, corrections to transverse inertia and stiffness associated with nonuniformity in pipe mass and bending stiffness distribution. Inertial fluid forces are represented by a Coriolis acceleration term $2 \alpha v \dot{u}^{\prime}$, arising due to pipe segments rotating at angular velocity $\dot{u}^{\prime}$, and a centripetal acceleration term $\alpha v^{2} u^{\prime \prime}$, accounting for the fluid with speed $v$ following a path with instantaneous curvature radius $\approx 1 / u^{\prime \prime}$; Similar terms occur in many pipe-flow studies (e.g. $[1,5,10,12]$ ). Initial pipe stretching and axially fixed supports introduces the two terms multiplied by $\mu^{2}$ : The first one, $\mu^{2} \eta$, is the axial tension force required to initially stretch the pipe, while the 
second one, with $\mu^{2}$ multiplying a nonlinear integrand, represents the additional axial force needed to stretch the pipe between immovable supports, at a given transverse pipe deformation $u$.

The nonlinear term $\gamma u^{2}$ represents asymmetric (w.r.t. $u=0$ ) stiffness, i.e. an elastic restoring force acting in the same direction regardless of the sign of the deformation; it could arise, e.g., from initial pipe curvature, or bias effects from pipe actuator magnetic coils, or from an opening and closing crack. Generalized velocity-dependent and uniformly distributed damping is included by the term $\beta f(\dot{u})$, where $f$ is an arbitrary nonlinear function, and $\beta$ a magnitude parameter.

The two stiffness type nonlinearities included are those supposed to be most influential for real Coriolis flowmeters. In the general case stiffness nonlinearities could arise from other sources, e.g. from a nonlinear curvature measure at large deflection slopes. However, Coriolis flowmeters are typically restrained at both pipe ends so as to prevent large curvatures; thus nonlinearity from midplane stretching limits the response so strongly that curvature nonlinearity remains insignificant in comparison. Furthermore, in the analysis to follow the nonlinear terms in the reduced system takes the form of just quadratic and cubical polynomial terms, thus rendering the analysis results applicable for any kind of nonlinearity that results in such terms, including e.g. curvature nonlinearity.

The smallness parameter $\varepsilon$ has no physical interpretation, but serves the purpose of magnitude bookkeeping through the different stages of analysis, explicitly quantifying the assumed order of magnitude of terms. In the final application of analysis results we just substitute for an $\varepsilon$ marked quantity (e.g. $\left.\varepsilon \mu^{2}\right)$ its specific value $\left(\mu^{2}\right)$, being reminded that for the results to be accurate the term with this parameter should be small compared to other terms in the equation. Note that an $\varepsilon$ in front of a parameter not necessarily indicates this parameter is small compared to unity, but that the entire term is small compared to other terms in the same equation. For example, the term in (1) with $\mu^{2}$ is multiplied by $\varepsilon$ and is thus assumed small, even if $\mu$ itself is assumed to exceed 200 (for a slender pipe). In this case the term is assumed small anyway, meaning that the total pipe stiffness is dominated by another term (here the bending stiffness $\left.u^{\prime \prime \prime}\right)$; in realistic examples this turns out as a large value of $\mu^{2}$ multiplying a very small integral with $\left(u^{\prime}\right)^{2}$, resulting in a small number.

\subsection{Physical assumptions detailed}

All system parameters are constant in time, or slowly varying so that any parameter $P$ changes only insignificantly during a period $2 \pi / \Omega$ of excitation, i.e. $|\dot{P} / P| 2 \pi / \Omega \ll 1$.

Transverse deflections in the plane of the excitation force are the dominating motions, with deflection slopes during vibrations being small, $(\partial \tilde{u} / \partial \tilde{x})^{2}=\left(u^{\prime}\right)^{2} \ll 1$, and the axial inertia small enough to be ignored. The pipe can be considered a slender beam structure $(\mu \tilde{\lambda} / l>200$,

where $\tilde{\lambda}$ is the shortest active vibration wavelength), so that shear deformations and rotary inertia can be ignored and Bernoulli-Euler beam theory employed. The pipe has only small axial variations in cross section, (integrated) density, and bending stiffness, and uniformly small mid-plane stretching. The transverse pipe stiffness is dominated by bending stiffness, with changes in transverse stiffness provided by pre-tension being small in comparison.

Longitudinal deformations $w=\tilde{w} / l$ satisfy the boundary conditions $w(0, t)=w(1, t)-\eta=0$, and are second in order as compared to transverse deflections, $w=O\left(u^{2}\right)$. Also, Hooke's law and a Cauchy measure of strain can be used when deriving an approximate expression for the effect of mid-plane stretching $[24,26]$. 
The damping terms with coefficients $c_{u}$ and $c_{\theta}$ are small, as are the terms with coefficients $k_{u}$ and $k_{\theta}$ defining additional nonuniform stiffness, the asymmetric stiffness $\gamma$, the uniform generalized damping $\beta f(\dot{u})$, and the external forcing amplitude $p$. The system is driven at resonance, but is not internally resonant; in particular the linear natural frequencies for the two lowest vibration modes are not close to being in ratio two or three.

Fluid flows inside the pipe from $x=0$ towards $x=1$ with a flat ("plug flow") velocity profile defined by a single constant velocity parameter $v$, and is assumed to be incompressible (measurement effects of non-flat profiles and flow compressibility were considered in $[38,39])$; this is justified when the local flow speed $|\mathbf{v}|$ is everywhere much smaller than the local speed of transverse elastic waves in the pipe material [40], and implies $\left(v m_{f}\right)^{\prime}=0$ so that $v^{\prime}=0$, and $0<v \ll 1$. Together with the assumption of small or no pipe pre-compression, this implies $u$ $=0$ is the only stable equilibrium for the un-actuated pipe $(p=0)$, and that axial loads are well below buckling values.

Consideration to gravity of a pipe hanging vertically in gravity would lead to additional terms in the equation of motion (see e.g. ( [4]); one can show that this corresponds to letting $k_{\theta}(x)=\tilde{g}^{2}(1-x)$ in the linear stiffness operator $L_{k}$ in (2), where $\tilde{g}=\sqrt{g / l} / \tilde{\omega}_{0}$ is a nondimensional measure of gravity $g$. This parameter can be interpreted as the ratio of two natural frequencies: $\sqrt{g / l}$, describing the order of magnitude of the frequency of rigid body pendulumlike oscillations of a fluid-filled pipe freely hanging in gravity, and $\tilde{\omega}_{0}$, describing the order of magnitude of structural bending vibrations of the pipe. For the applications of interest here this ratio is naturally very small, and gravity is thus ignored in comparison with other forces present.

All of the above assumptions hold approximately for applications such as Coriolis flowmetering under typical operating conditions. The major factor ignored as compared to real industrial flowmeters is their more complicated geometry (many have two curved pipes rather than one straight). This allows for simple analytical expressions giving direct insight into the various effects under study, and may form the basis for posing testable hypotheses for real flowmeters.

\subsection{Pipe perfectness}

For this study we define a perfect pipe as being undamped, with axially uniform cross section, density, and linear bending stiffness, zero mass flow, and no additional transverse stiffness (by pre-tension, midplane stretching, or other). This implies that for a perfect pipe $\Delta m=\alpha v=\Delta e$ $=\mu=\eta=\gamma=L_{k}=L_{c}=\beta=0$, so that in the left-hand side of (1) the entire bracketed term vanishes. (Actually $\mu$ is not zero for the perfect pipe, but the parenthesis it multiplies in (1) is; letting $\mu=0$ has the same effect and is notationally more convenient.) Thus an imperfect pipe is considered and treated mathematically as a small perturbation of a perfect pipe, in that one or more of the imperfection parameters or functions are nonzero, and their corresponding term in (1) is nonzero, but small.

Next we employ perturbation analysis to calculate the dynamic response of the imperfect pipe, aiming at insight into how each imperfection affects Coriolis flowmeter (model) performance. 


\section{Primary resonant response: Analytical prediction using perturbation analysis}

\subsection{Method}

The equation of motion (1) with (2)-(4), describing the continuous fluid conveying pipe, is a nonlinear partial differential equation with homogeneous boundary conditions. The following analysis method was suggested in [1] for a similar problem, and used subsequently also in $[18,29]$; here it will be employed in a slightly simpler version, by introducing Galerkin-expansion at an earlier stage of analysis, like in [10]. A solution $u(x, t)$ is sought that is approximately valid under the assumptions stated in Sect. 2.4, and which can be used for setting up a simple analytical prediction for the difference $\Delta \psi$ in vibration phase measured between two pipe points symmetrically situated about $x=\frac{1}{2}$. This phase shift, or the corresponding time shift in velocity zero-crossing, is the quantity actually measured in Coriolis flowmetering. For manufacturers it is important to be able to predict how the measured phase shift depends on factors other than mass flow, e.g. parameters describing nonuniformity, asymmetry, and other imperfections.

The aim is here at transparent analytical expressions, allowing for direct insight into the effects of primary physical parameters on amplitude, phase and especially phase shift. Solutions of the equation of motion are approximated by a Galerkin expansion in the first (symmetric) and the second (antisymmetric) linear pipe mode, which are those of primary importance for Coriolis flowmetering. Time dependent modal amplitude functions are then approximated using a systematic perturbation approach, and used to calculate corresponding approximate analytical predictions for vibration amplitudes and phase shift. Important results can be inferred directly by inspecting the analytical expressions. Their mathematical accuracy is tracked using order symbols, and checked later (Sect. 5) by comparing to results of direct numerical simulation of (1).

\subsection{Galerkin discretization and approximation}

With pipe imperfections assumed small, it is reasonable to use mode shapes for the perfect pipe as expansion functions. These are obtained by solving (1) in its unperturbed form $(\varepsilon=0)$. Inserting a solution $u(x, t)=\varphi(x) \sin (\omega t+\psi)$ gives $\varphi^{\prime \prime \prime \prime}=\omega^{2} \varphi$, which with boundary conditions (3) or (4) constitutes a standard differential eigenvalue problem. Its solution gives the natural frequencies $[26] \omega=\omega_{j}, j=1,2, \cdots$, :

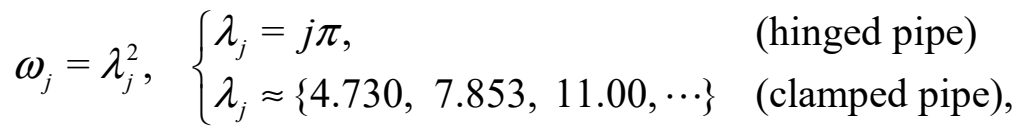

and corresponding mode shapes:

$$
\varphi_{j}(x)= \begin{cases}\sqrt{2} \sin \left(\lambda_{j} x\right), & \text { (hinged) } \\ \cosh \left(\lambda_{j} x\right)-\cos \left(\lambda_{j} x\right)-\frac{\cosh \lambda_{j}-\cos \lambda_{j}}{\sinh \lambda_{j}-\sin \lambda_{j}}\left(\sinh \left(\lambda_{j} x\right)-\sin \left(\lambda_{j} x\right)\right) & \text { (clamped) }\end{cases}
$$

which are orthogonal on $x \in[0,1]$, and unit-normalized so that

$$
\int_{0}^{1} \varphi_{i} \varphi_{j} \mathrm{~d} x=\omega_{j}^{-2} \int_{0}^{1} \varphi_{i} \varphi_{j}^{\prime \prime \prime \prime} \mathrm{d} x=\omega_{j}^{-2} \int_{0}^{1} \varphi_{i}^{\prime \prime} \varphi_{j}^{\prime \prime} \mathrm{d} x=\delta_{i j},
$$


where $\delta_{i j}$ is the Kronecker delta. For the hinged-hinged and clamped-clamped supports considered in this study, the odd modes are symmetric w.r.t. $x=\frac{1}{2}$, while the even modes are antisymmetric, i.e. $\varphi_{2 j-1}(x)=\varphi_{2 j-1}(1-x)$ and $\varphi_{2 j}(x)=-\varphi_{2 j}(1-x)$ for $j=1,2, \ldots$.

As an $N$-term Galerkin-expansion [41] for the solution to (1) in the presence of imperfections $(\varepsilon \neq 0)$ we then take:

$$
u(x, t)=\sum_{j=1}^{N} q_{j}(t) \varphi_{j}(x)
$$

approaching the exact solution as $N \rightarrow \infty$, where $q_{j}(t)$ are the time-varying modal amplitudes to be determined. Inserting (9) into (1), multiplying by $\varphi_{i}$, and integrating over the pipe length gives a set of modal equations of motion:

$$
\begin{aligned}
\ddot{q}_{i}+\omega_{i}^{2} q_{i}+\varepsilon\left\{\sum_{j=1}^{N}\left(m_{i j} \ddot{q}_{j}+c_{i j} \dot{q}_{j}+\left(k_{i j}+n_{i j}\right) q_{j}+\alpha\left(v^{2} B_{i j} q_{j}+2 v C_{i j} \dot{q}_{j}\right)\right)\right. \\
\left.\quad-\mu^{2}\left(\eta-\frac{1}{2} \sum_{r, s=1}^{N} B_{r s} q_{r} q_{s}\right) \sum_{j=1}^{N} B_{i j} q_{j}+\gamma \sum_{j, k=1}^{N} H_{i j k} q_{j} q_{k}+\beta \int_{0}^{1} \varphi_{i} f\left(\sum_{j=1}^{N} \dot{q}_{j} \varphi_{j}\right) \mathrm{d} x\right\} \\
\quad=\varepsilon p \cos (\Omega t) \varphi_{i}\left(x_{p}\right), \quad i=1,2, \ldots, N .
\end{aligned}
$$

where the orthogonality properties (8) of the normalized modes shapes (7) has been employed, constants in uppercase define integrals depending only on mode shapes:

$$
B_{i j}=\int_{0}^{1} \varphi_{i} \varphi_{j}^{\prime \prime} \mathrm{d} x=-\int_{0}^{1} \varphi_{i}^{\prime} \varphi_{j}^{\prime} \mathrm{d} x, \quad C_{i j}=\int_{0}^{1} \varphi_{i} \varphi_{j}^{\prime} \mathrm{d} x, \quad H_{i j k}=\int_{0}^{1} \varphi_{i} \varphi_{j} \varphi_{k} \mathrm{~d} x,
$$

constants in lowercase define mode shape integrals weighted by imperfection functions:

$$
\begin{aligned}
& m_{i j}=\int_{0}^{1} \Delta m(x) \varphi_{i} \varphi_{j} \mathrm{~d} x, \\
& n_{i j}=\int_{0}^{1} \varphi_{i}\left(\Delta e(x) \varphi_{j}^{\prime \prime}\right)^{\prime \prime} \mathrm{d} x=\int_{0}^{1} \Delta e(x) \varphi_{i}^{\prime \prime} \varphi_{j}^{\prime \prime} \mathrm{d} x, \\
& k_{i j}=\int_{0}^{1} \varphi_{i} L_{k}\left[\varphi_{j}\right] \mathrm{d} x=\int_{0}^{1} k_{u}(x) \varphi_{i} \varphi_{j} \mathrm{~d} x+\int_{0}^{1} k_{\theta}(x) \varphi_{i}^{\prime} \varphi_{j}^{\prime} \mathrm{d} x, \\
& c_{i j}=\int_{0}^{1} \varphi_{i} L_{c}\left[\varphi_{j}\right] \mathrm{d} x=\int_{0}^{1} c_{u}(x) \varphi_{i} \varphi_{j} \mathrm{~d} x+\int_{0}^{1} c_{\theta}(x) \varphi_{i}^{\prime} \varphi_{j}^{\prime} \mathrm{d} x,
\end{aligned}
$$

and for the hinged-hinged or clamped-clamped supports considered in this study:

$$
B_{i j}=0 \text { for } i+j \text { odd, } C_{i j}=0 \text { for } i+j \text { even, } H_{i j k}=0 \text { for } i+j+k \text { even. }
$$

The pipe is assumed to be resonantly excited at the fundamental symmetric mode $\varphi_{1}$ by external actuation at frequency $\Omega \approx \omega_{1}$; the fluid flow then induces asymmetric (w.r.t. $x=\frac{1}{2}$ ) Coriolis forces, which excite the second, antisymmetric pipe mode $\varphi_{2}$, though still (mainly) at the frequency $\omega_{1}$. The result is a traveling elastic wave of transverse pipe motion, whose phase shift along the pipe in Coriolis flowmeters is picked up by motion sensors and used to estimate masse flow. The main effect can thus be expected to be well estimated by including just the first two terms of the Galerkin expansion (9).

The corresponding pair of coupled ordinary differential equations governing the modal amplitudes $q_{1}(t)$ and $q_{2}(t)$ are obtained by letting $N=2$ in (10) and rearranging into: 


$$
\begin{aligned}
\ddot{q}_{i}+\omega_{i}^{2} q_{i}+\varepsilon\{ & \left\{m_{i 1} \ddot{q}_{1}+m_{i 2} \ddot{q}_{2}+\hat{c}_{i 1} \dot{q}_{1}+\hat{c}_{i 2} \dot{q}_{2}+K_{i 1} q_{1}+K_{i 2} q_{2}\right. \\
& +\gamma\left(H_{i 11} q_{1}^{2}+H_{i 22} q_{2}^{2}+2 H_{i 12} q_{1} q_{2}\right) \\
& +\frac{1}{2} \mu^{2}\left(B_{i 1}{ }^{2} q_{1}^{3}+B_{i 2}{ }^{2} q_{2}^{3}+B_{i 1} B_{22} q_{1} q_{2}^{2}+B_{11} B_{i 2} q_{1}^{2} q_{2}\right) \\
& \left.+\beta \int_{0}^{1} \varphi_{i} f\left(\dot{q}_{1} \varphi_{1}+\dot{q}_{2} \varphi_{2}\right) \mathrm{d} x\right\}=\varepsilon p \cos (\Omega t) \varphi_{i}\left(x_{p}\right), \quad i=1,2,
\end{aligned}
$$

where new stiffness and damping parameters have been introduced, respectively:

$$
\begin{aligned}
& K_{i j}=k_{i j}+n_{i j}+\left(\alpha v^{2}-\mu^{2} \eta\right) B_{i j}, \\
& \hat{c}_{i j}=c_{i j}+2 \alpha v C_{i j} .
\end{aligned}
$$

Here the mass flow $\alpha v$ appears also in the damping-like terms $\hat{c}_{j k} \dot{q}_{k}$, but these are not purely dissipative: Since in general $C_{i j} \neq C_{j i}$ (cf. (11)), the corresponding linear damping matrix with components $\hat{c}_{i j}$ has an antisymmetric part with components $\frac{1}{2}\left(\hat{c}_{i j}-\hat{c}_{j i}\right)$, corresponding to the gyroscopic forces [42] associated with the Coriolis term $2 \alpha v \dot{u}^{\prime}$ in (1); these forces are conservative. (This holds only with identical boundary conditions at the pipe ends; with e.g. clamped-free boundaries the matrix with components $C_{i j}$ will have also symmetric components, implying the Coriolis forces would be nonconservative. The effect of this on vibration phase shifts could be of technical interest for certain applications, though maybe less so for Coriolis flowmetering.)

The two-mode approximation for transverse motions $u(x, t)$ of the pipe is then given by (9) with $N=2, \varphi_{1}(x)$ and $\varphi_{2}(x)$ given by (7), and $q_{1}(t)$ and $q_{2}(t)$ by the solutions to (14), to be calculated next.

\subsection{Approximate solution using perturbation analysis}

With $\varepsilon \ll 1$ perturbation analysis can be used to calculate approximate solutions to (14). Using the method of multiple scales $[24,43,44]$ we seek a solution in the form:

$$
\begin{aligned}
& q_{1}=q_{10}\left(T_{0}, T_{1}\right)+\varepsilon q_{11}\left(T_{0}, T_{1}\right)+O\left(\varepsilon^{2}\right) \\
& q_{2}=\varepsilon q_{21}\left(T_{0}, T_{1}\right)+O\left(\varepsilon^{2}\right),
\end{aligned}
$$

where $T_{0}=t, T_{1}=\varepsilon t$ is the slow timescale, and $O\left(\varepsilon^{n}\right)$ denotes terms of order of magnitude $\varepsilon^{n}$ and smaller. The omission of an $\mathcal{E}^{0}$-term in the expansion for $q_{2}$ simplifies the calculation, and is readily justified by a known property of the solutions sought: In Coriolis flowmetering the fundamental mode $\varphi_{1}$ is resonantly excited and thus its amplitude $q_{1}$ dominates the modal response; the second mode $\varphi_{2}$ is not resonantly excited, but activated only by the small nonidealities of the system (e.g. Coriolis forces from the flow), thus $\left|q_{2}\right| \ll\left|q_{1}\right|$, as is reflected in (16).

Inserting (16) into the two equations in (14) and balancing terms of like powers of $\varepsilon$, one obtains from the $\varepsilon^{0}$-terms an equation for the dominating amplitude component $q_{10}$ of the fundamental modal amplitude $q_{1}$ :

$$
\mathrm{D}_{0}^{2} q_{10}+\omega_{1}^{2} q_{10}=0
$$

where $\mathrm{D}_{i}^{j} \equiv \partial^{j} / \partial T_{i}^{j}$. Similarly, the $\mathcal{E}^{1}$-terms give for $j=1$ an equation for the small amplitude correction $q_{11}$ to the fundamental mode: 


$$
\begin{aligned}
\mathrm{D}_{0}^{2} q_{11}+\omega_{1}^{2} q_{11}= & -2 \mathrm{D}_{0} \mathrm{D}_{1} q_{10}-m_{11} \mathrm{D}_{0}^{2} q_{10}-K_{11} q_{10}-\hat{c}_{11} \mathrm{D}_{0} q_{10}-\frac{1}{2} \mu^{2} B_{11}^{2} q_{10}^{3} \\
& -\gamma H_{111} q_{10}^{2}-\beta \int_{0}^{1} \varphi_{1} f\left(\mathrm{D}_{0} q_{10} \varphi_{1}\right) \mathrm{d} x+p \varphi_{1}\left(x_{p}\right) \cos \left(\Omega T_{0}\right),
\end{aligned}
$$

and for $j=2$ an equation for the small amplitude $q_{21}$ of the second mode:

$$
\begin{aligned}
\mathrm{D}_{0}^{2} q_{21}+\omega_{2}^{2} q_{21}= & -m_{21} \mathrm{D}_{0}^{2} q_{10}-K_{21} q_{10}-\hat{c}_{21} \mathrm{D}_{0} q_{10} \\
& -\beta \int_{0}^{1} \varphi_{2} f\left(\mathrm{D}_{0} q_{10} \varphi_{1}\right) \mathrm{d} x+p \varphi_{2}\left(x_{p}\right) \cos \left(\Omega T_{0}\right),
\end{aligned}
$$

where, according to (15):

$$
K_{11}=k_{11}+n_{11}+\left(\alpha v^{2}-\mu^{2} \eta\right) B_{11}, \quad K_{21}=k_{21}+n_{21}, \quad \hat{c}_{11}=c_{11}, \quad \hat{c}_{21}=c_{21}+2 \alpha \nu C_{21},
$$

and (13) has been employed in (18)-(20).

Equation (17) is a second order linear partial differential equation with a solution:

$$
q_{10}=A\left(T_{1}\right) \mathrm{e}^{\mathrm{i} \omega_{1} T_{0}}+\text { c.c. }
$$

where $A\left(T_{1}\right)$ is a complex-valued function of the slow timescale only, $\mathrm{i}$ is the imaginary unit and c.c. here and below denotes complex conjugates of all preceding terms.

As appears from (21) the function $q_{10}$ is $2 \pi$-periodic in $\omega_{1} T_{0}$, and so will be the argument to the general damping function $f$ in (18)-(19). Then $f$ is also $2 \pi$-periodic in $\omega_{1} T_{0}$, and can thus be Fourier-expanded, with (21) substituted into the argument of $f$ :

$$
f\left(\mathrm{i} \omega_{1} A \mathrm{e}^{\mathrm{i} \omega_{1} T_{0}} \varphi_{1}+\text { c.c. }\right)=\frac{1}{2} g_{0}+\sum_{n=1}^{\infty} g_{n} \mathrm{e}^{\mathrm{i} n \omega_{1} T_{0}}+\text { c.c., }
$$

where $g_{n}$ is the $n$ 'th Fourier coefficient:

$$
g_{n}\left(A\left(T_{1}\right), \varphi_{1}(x)\right)=\frac{\omega_{1}}{2 \pi} \int_{0}^{2 \pi / \omega_{1}} f\left(\mathrm{i} \omega_{1} A \mathrm{e}^{\mathrm{i} \omega_{1} T_{0}} \varphi_{1}+\text { c.c. }\right) \mathrm{e}^{-\mathrm{i} n \omega_{1} T_{0}} \mathrm{~d} T_{0}, \quad n=0,1, \ldots
$$

Inserting (21)-(22) into (18)-(19) then gives:

$$
\begin{aligned}
\mathrm{D}_{0}^{2} q_{11} & +\omega_{1}^{2} q_{11}=-\gamma H_{111} A \bar{A}-\gamma H_{111} A^{2} \mathrm{e}^{\mathrm{i} 2 \omega_{1} T_{0}}-\frac{1}{2} \mu^{2} B_{11}^{2} A^{3} \mathrm{e}^{\mathrm{i} 3 \omega_{1} T_{0}} \\
+ & {\left[\left(\omega_{1}^{2} m_{11}-K_{11}-\mathrm{i} \omega_{1} \alpha_{11}-\frac{3}{2} \mu^{2} B_{11}^{2} A \bar{A}\right) A-\mathrm{i} 2 \omega_{1} \mathrm{D}_{1} A+\frac{1}{2} p \varphi_{1}\left(x_{p}\right) \mathrm{e}^{\mathrm{i} \sigma T_{1}}\right] \mathrm{e}^{\mathrm{i} \omega_{1} T_{0}} } \\
- & \beta \int_{0}^{1} \varphi_{1}\left(\frac{1}{2} g_{0}+\sum_{n=1}^{\infty} g_{n} \mathrm{e}^{\mathrm{i} \omega_{1} T_{0}}\right) \mathrm{d} x+\text { c.c. },
\end{aligned}
$$

and

$$
\begin{aligned}
\mathrm{D}_{0}^{2} q_{21}+\omega_{2}^{2} q_{21}= & {\left[\left(\omega_{1}^{2} m_{21}-K_{21}-\mathrm{i} \omega_{1} \alpha_{21}\right) A+\frac{1}{2} p \varphi_{2}\left(x_{p}\right) \mathrm{e}^{\mathrm{i} \sigma T_{1}}\right] \mathrm{e}^{\mathrm{i} \omega_{1} T_{0}} } \\
& -\beta \int_{0}^{1} \varphi_{2}\left(\frac{1}{2} g_{0}+\sum_{n=1}^{\infty} g_{n} \mathrm{e}^{\mathrm{i} n \omega_{1} T_{0}}\right) \mathrm{d} x+\text { c.c. },
\end{aligned}
$$

where a detuning parameter $\sigma$ has been introduced to express the nearness to first-mode primary resonance, i.e. the nearness of the excitation frequency $\Omega$ to the fundamental natural frequency $\omega_{1}$ of the perfect pipe :

$$
\Omega=\omega_{1}+\varepsilon \sigma \quad\left(\Rightarrow \Omega T_{0}=\omega_{1} T_{0}+\sigma T_{1}\right)
$$


The requirement for solutions $q_{11}$ of (24) to be free of secular terms is that the resonant excitation terms (proportional to $\mathrm{e}^{\mathrm{i} \omega_{1} T_{0}}$ ) vanish identically, i.e. the solvability condition becomes:

$$
\left(\omega_{1}^{2} m_{11}-K_{11}-\mathrm{i} \omega_{1} \hat{c}_{11}-\frac{3}{2} \mu^{2} B_{11}^{2} A \bar{A}\right) A-\mathrm{i} 2 \omega_{1} \mathrm{D}_{1} A+\frac{1}{2} p \varphi_{1}\left(x_{p}\right) \mathrm{e}^{\mathrm{i} \sigma T_{1}}-\beta \int_{0}^{1} \varphi_{1} g_{1} \mathrm{~d} x=0 .
$$

With this fulfilled, a particular solution of (24) is:

$$
\begin{aligned}
& q_{11}=-\frac{1}{\omega_{1}^{2}}[(\left.\gamma H_{111}\left(\bar{A}+\frac{1}{3} A \mathrm{e}^{\mathrm{i} 2 \omega_{1} T_{0}}\right) A+\frac{1}{16} \mu^{2} B_{11}^{2} A^{3} \mathrm{e}^{\mathrm{i} 3 \omega_{1} T_{0}}\right) \\
&\left.+\beta\left(\int_{0}^{1} \varphi_{1}\left(\frac{1}{2} g_{0}+\sum_{n=2}^{\infty}\left(1-n^{2}\right)^{-1} g_{n} e^{\mathrm{i} n \omega_{1} T_{0}}\right) \mathrm{d} x\right)\right]+ \text { c.c. }
\end{aligned}
$$

Similarly, for solutions $q_{21}$ (25) to be free of secular terms, excitation terms proportional to $e^{i \omega_{2} T_{0}}$ should vanish identically. Assuming $\omega_{2}$ is away from $\omega_{1}$ there will be no such terms, unless the damping is nonlinear $(\beta \neq 0)$, and at the same time an internal resonance exist between the lowest to natural frequencies of the perfect pipe, i.e. $\omega_{2} \approx n \omega_{1}$; this is the well-known case of modal interaction [24,26], where nonlinearity and internal resonance combines to allow the transfer of energy from a directly excited mode (here $\varphi_{1}$ ) to another mode (here $\varphi_{2}$ ). Such near-integer relationships between the lowest natural frequencies could cause anomalies in the functioning of Coriolis flowmeters, in the presence of nonlinearity, since the second mode would be excited not only by the fluid flow. For this present study we assume the pipe is designed so as to not possess internal resonance. A particular solution to (25) is then:

$$
\begin{aligned}
q_{21}= & \frac{1}{\omega_{2}^{2}-\omega_{1}^{2}}\left[\left(\omega_{1}^{2} m_{21}-K_{21}-\mathrm{i} \omega_{1} \hat{c}_{21}\right) A+\frac{1}{2} p \varphi_{2}\left(x_{p}\right) \mathrm{e}^{\mathrm{i} \sigma T_{1}}\right] \mathrm{e}^{\mathrm{i} \omega_{1} T_{0}} \\
& -\frac{\beta}{2 \omega_{2}^{2}} \int_{0}^{1} \varphi_{2}\left(g_{0}+\sum_{n=1}^{\infty} \frac{2 g_{n} \mathrm{e}^{\mathrm{i} n \omega_{1} T_{0}}}{1-\left(n \omega_{1} / \omega_{2}\right)^{2}}\right) \mathrm{d} x+\text { c.c. }
\end{aligned}
$$

To determine the amplitude function $A\left(T_{1}\right)$ from (27) we express it in polar form,

$$
A\left(T_{1}\right)=\frac{1}{2} a\left(T_{1}\right) \mathrm{e}^{\mathrm{i} \phi\left(T_{1}\right)},
$$

where $a$ and $\phi$ are real-valued functions to be determined. In terms of these, the Fourier coefficients (23) can be written

$$
g_{n}=\left(\kappa_{n}-\mathrm{i} \xi_{n}\right) e^{\mathrm{i} n \phi}, n=0,1, \ldots
$$

where

$$
\begin{aligned}
& \kappa_{n}=\kappa_{n}(x, a)=\frac{1}{2 \pi} \int_{0}^{2 \pi} f\left(-\omega_{1} a \varphi_{1}(x) \sin Y\right) \cos (n Y) \mathrm{d} Y, \\
& \xi_{n}=\xi_{n}(x, a)=\frac{1}{2 \pi} \int_{0}^{2 \pi} f\left(-\omega_{1} a \varphi_{1}(x) \sin Y\right) \sin (n Y) \mathrm{d} Y .
\end{aligned}
$$

One can show that, for any function $f$, (32) gives $\kappa_{n}=0$ for odd $n$ while $\xi_{n}=0$ for even $n$. Also, with $\varphi_{1}(x)$ being symmetric w.r.t. $x=\frac{1}{2}, \kappa_{n}$ and $\xi_{n}$ are symmetric w.r.t. $x$ as well. Finally, if $f$ is antisymmetric w.r.t. its argument, i.e. $f(-\dot{u})=-f(\dot{u})$ (as e.g. with damping functions of only odd-ordered powers of velocity), then $\kappa_{n}=0$ for all $n$, while if $f$ is symmetric, $f(-\dot{u})=f(\dot{u})$, then $\xi_{n}=0$ for all $n$. 
Inserting this and (30) into the solvability condition (27) gives, when multiplying by $\mathrm{e}^{-\mathrm{i} \phi}$ and separating real and imaginary parts, the autonomous modulation equations:

$$
\begin{gathered}
a^{\prime}=-\frac{1}{2} \hat{c}_{11} a+\frac{1}{2 \omega_{1}}\left[2 \beta \int_{0}^{1} \varphi_{1} \xi_{1} \mathrm{~d} x+p \varphi_{1}\left(x_{p}\right) \sin (\psi)\right], \\
a \psi^{\prime}=\sigma a+\frac{1}{2 \omega_{1}}\left[\left(\omega_{1}^{2} m_{11}-K_{11}-\frac{3}{8} \mu^{2} B_{11}^{2} a^{2}\right) a+p \varphi_{1}\left(x_{p}\right) \cos (\psi)\right],
\end{gathered}
$$

where ()$^{\prime} \equiv \mathrm{d} / \mathrm{d} T_{1}$, and a new phase variable has been introduced:

$$
\psi\left(T_{1}\right)=\sigma T_{1}-\phi\left(T_{1}\right),
$$

Inserting (30) also into (21) gives, with (26), and back substituting $T_{0}=t$ and $T_{1}=\varepsilon t$ :

$$
q_{10}=\frac{1}{2} a \mathrm{e}^{\mathrm{i}\left(\omega_{1} T_{0}+\phi\right)}+\text { c.c. }=a \cos \left(\omega_{1} T_{0}+\sigma T_{1}-\psi\right)=a \cos (\Omega t-\psi),
$$

while inserting (30) into (28)-(29) with similar back substitutions gives:

$$
\begin{aligned}
q_{11}= & -\frac{1}{\omega_{1}^{2}}\left[\frac{1}{2} \gamma H_{111}\left(1+\frac{1}{3} \cos (2(\Omega t-\psi))\right) a^{2}+\frac{1}{64} \mu^{2} B_{11}^{2} a^{3} \cos (3(\Omega t-\psi))\right] \\
& -\frac{\beta}{\omega_{1}^{2}} \int_{0}^{1} \varphi_{1}\left(\kappa_{0}-2 \sum_{n=2}^{\infty} \frac{\kappa_{n} \cos (n(\Omega t-\psi))+\xi_{n} \sin (n(\Omega t-\psi))}{n^{2}-1}\right) \mathrm{d} x,
\end{aligned}
$$

and:

$$
\begin{aligned}
q_{21}= & \frac{1}{\omega_{2}^{2}-\omega_{1}^{2}}\left[\left(\omega_{1}^{2} m_{21}-K_{21}\right) a \cos (\Omega t-\psi)+\omega_{1} \hat{c}_{21} a \sin (\Omega t-\psi)+p \varphi_{2}\left(x_{p}\right) \cos (\Omega t)\right] \\
& -\frac{\beta}{\omega_{2}^{2}} \int_{0}^{1} \varphi_{2}\left(\kappa_{0}-2 \sum_{n=1}^{\infty} \frac{\kappa_{n} \cos (n(\Omega t-\psi))+\xi_{n} \sin (n(\Omega t-\psi))}{\left(n \omega_{1} / \omega_{2}\right)^{2}-1}\right) \mathrm{d} x,
\end{aligned}
$$

The two-mode approximate perturbation solution for the transverse pipe vibrations $u(x, t)$ is then obtained from (9) with (16) and (36) inserted:

$$
u(x, t)=a(t) \cos (\Omega t-\psi(t)) \varphi_{1}(x)+\varepsilon\left[q_{11}(t) \varphi_{1}(x)+q_{21}(t) \varphi_{2}(x)\right]+O\left(\varepsilon^{2}\right),
$$

where $q_{11}(t)$ and $q_{21}(t)$ are given by (37)-(38) and the slowly varying amplitude $a(t)$ and phase $\psi(t)$ are solutions to the modulation equations (33)-(34).

For flowmeter applications we are in particular interested in the stationary vibrations that remain when transients caused by disturbances of any kind (e.g. a change in flow speed or actuator force) have damped away. The corresponding stationary solutions to (33)-(34) are characterized by having constant amplitude $a(t)=\hat{a}$ and phase $\psi(t)=\hat{\psi}$, as determined by letting $a^{\prime}=\psi^{\prime}=0$ in (33)-(34) and solving the resulting algebraic pair of equations for $\hat{a}$ and $\hat{\psi}$. Eliminating $\hat{\psi}$ from the two equations so obtained, and inserting (26), gives the algebraic frequency response equation, implicitly defining the relation between the stationary vibration amplitude $\hat{a}$ and the excitation frequency $\Omega$ :

$$
\begin{aligned}
& \left(K_{11}-\omega_{1}^{2} m_{11}-2 \omega_{1}\left(\Omega-\omega_{1}\right)+\frac{3}{8} \mu^{2} B_{11}^{2} \hat{a}^{2}\right)^{2} \hat{a}^{2} \\
+ & \left(\omega_{1} \hat{c}_{11} \hat{a}-2 \beta \int_{0}^{1} \varphi_{1}(x) \xi_{1}(x, \hat{a}) \mathrm{d} x\right)^{2}=p^{2} \varphi_{1}^{2}\left(x_{p}\right)
\end{aligned}
$$


where the three groups of terms represent, respectively, (dynamic) stiffness, dissipation, and forced excitation. Dividing the first of the two aforementioned equations ((33)-(34) with $a^{\prime}=\psi^{\prime}=0$ ) with the second gives the corresponding stationary phase $\hat{\psi}$ :

$$
\tan \hat{\psi}=\frac{\omega_{1} \hat{c}_{11} \hat{a}-2 \beta \int_{0}^{1} \varphi_{1}(x) \xi_{1}(x, \hat{a}) \mathrm{d} x}{\left(K_{11}-\omega_{1}^{2} m_{11}-2 \omega_{1}\left(\Omega-\omega_{1}\right)+\frac{3}{8} \mu^{2} B_{11}^{2} \hat{a}^{2}\right) \hat{a}},
$$

where the numerator represents energy dissipation and the denominator dynamic stiffness (vanishing at resonance).

The frequency response curve $(\Omega, \hat{a})$ can be described by solving (40) for $\Omega$, giving:

$$
\Omega=\hat{\omega}_{1}+\frac{3 \mu^{2} B_{11}^{2}}{16 \omega_{1}} \hat{a}^{2} \pm \frac{1}{2} \sqrt{\left(\frac{p \varphi_{1}\left(x_{p}\right)}{\omega_{1} \hat{a}}\right)^{2}-\left(\hat{c}_{11}-\frac{2 \beta}{\omega_{1} \hat{a}} \int_{0}^{1} \varphi_{1}(x) \xi_{1}(x, \hat{a}) \mathrm{d} x\right)^{2}},
$$

where

$$
\hat{\omega}_{1}=\left(1-\frac{1}{2} m_{11}\right) \omega_{1}+\frac{K_{11}}{2 \omega_{1}}
$$

is the linear $\left(\mu^{2}=0\right.$ or $\left.\hat{a}^{2} \ll 1\right)$ natural frequency $\hat{\omega}_{1}$ of the pipe in the presence of nonuniform pipe mass $\left(m_{11} \neq 0\right)$ and any factor causing $K_{11} \neq 0$ (i.e. additional translational stiffness $L_{k}$, bending stiffness nonuniformity $\Delta e$, mass flow $\alpha v$, or axial tension $\mu^{2} \eta$ ).

The frequency response is qualitatively illustrated in Fig. 2, for a case where the effect of stretching nonlinearity (second term in (42)) is sufficiently strong to create a frequency "overhang" region where two stable vibration amplitudes $\hat{a}$ exist. The two first terms of (42) defines the backbone of the frequency response (dash-dotted in Fig. 2), with the basic relationship between frequency and amplitude for free oscillations (undamped and unforced):

$$
\Omega=\hat{\omega}_{1}+\frac{3 \mu^{2} B_{11}^{2}}{16 \omega_{1}} \hat{a}^{2} \text { for the backbone }\left(p=\hat{c}_{11}=\beta=0\right),
$$

which grows quadratically with vibration amplitude $\hat{a}$ from the linear natural frequency $\hat{\omega}_{1}$.

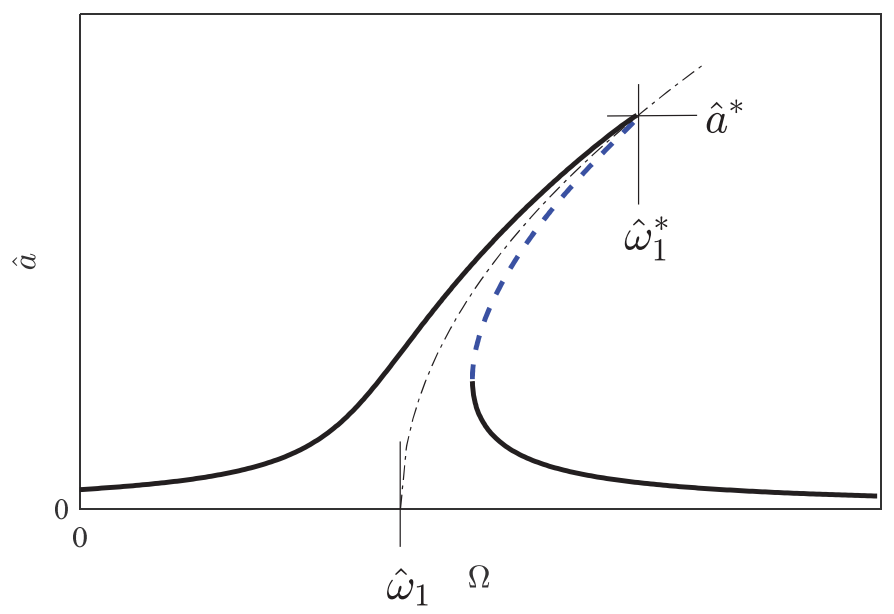

Fig. 2 Nonlinear frequency response according to (42): Stationary pipe vibration amplitude $\hat{a}$ of the fundamental harmonic as a function of excitation frequency $\Omega$. Stable (solid line), unstable (dashed), and free oscillation / backbone response (dash-dotted) 
The maximum forced response amplitude $\hat{a}=\hat{a}^{*}$ occurs where the width of the resonance peak vanishes (cf. Fig. 2). Equating to zero the radical in (42), and using (15) and (13) to obtain $\hat{c}_{11}=c_{11}$, this gives:

$$
\hat{a}^{*}=\frac{1}{c_{11} \omega_{1}}\left(p \varphi_{1}\left(x_{p}\right)+2 \beta \int_{0}^{1} \varphi_{1}(x) \xi_{1}\left(x, \hat{a}^{*}\right) \mathrm{d} x\right) .
$$

The corresponding peak frequency $\Omega=\hat{\omega}_{1}^{*}$ (see Fig. 2) is obtained from (42) or (44) with $\hat{a}=\hat{a}^{*}$ inserted:

$$
\hat{\omega}_{1}^{*}=\hat{\omega}_{1}+\frac{3}{16} \mu^{2} B_{11}^{2} \hat{a}^{* 2} / \omega_{1} .
$$

As appears from (45)-(46) the maximum amplitude is independent on the midplane stretching nonlinearity parameter $\mu$, which affects only the frequency at which the maximum amplitude occurs. As for the asymmetric stiffness nonlinearity parameter $\gamma$, it appears to affect neither the maximum amplitude or the frequency at which this occurs. However, besides the linear damping $\left(c_{11}\right)$, nonlinear damping $(\beta \neq 0)$ appears to affect the maximum amplitude, and also makes (45) nonlinear in $\hat{a}^{*}$.

With Coriolis flowmetering the pipe is automatically (by a positive velocity-feedback loop corresponding to negative damping) driven at the current resonance frequency, i.e. $\Omega=\hat{\omega}_{1}^{*}$, which changes slightly with mass flow $\alpha v$ via its influence on $K_{11}$, cf. (46), (43), and (15). As for the pipe vibration amplitude at this frequency, with stronger nonlinearity there is a theoretical risk that the stationary vibrations settle at the lower-amplitude stable branch of the frequency response, rather than at the peak. This could be avoided by design changes that alter the quantities $\mu, B_{11}$, and $\omega_{1}$ in the second term on the right-hand side of (46) and (42) sufficiently, that is: so that for any $\Omega$, Equation (42) has at most a single solution $\hat{a}$.

\subsection{Interpreting the general solution}

Equation (39), with $q_{11}$ and $q_{21}$ given by (37)-(38), shows that the pipe basically vibrates at amplitude $a$ in its driven fundamental mode $\varphi_{1}(x)$. On top of this are small additional motions of order $\varepsilon$, in both the first and the second vibration mode, accounting for the effects of mass flow $\alpha v$, for possible external excitation of the second mode $p \varphi_{2}\left(x_{p}\right)$, and for the various nonuniformities considered.

The first-mode correction amplitude $q_{11}$ in (39), as given by (37), vanishes identically if nonlinearities are ignorable $\left(\gamma=\mu^{2}=\beta=0\right)$, and can have a nonzero time average if $\gamma H_{111} \neq 0$ (i.e. with asymmetric stiffness, e.g. a pre-deformed pipe), or if $\kappa_{0} \neq 0$ (i.e. with asymmetric generalized damping functions $f(-\dot{u}) \neq-f(\dot{u}))$. Nonlinearity from asymmetric stiffness and midplane stretching ( $\gamma$ and $\mu^{2}$ terms) creates response components oscillating at multiples of the forcing frequency, as is typical for nonlinear systems. Such higher harmonics also arise with the $\beta$-term in (37), originating from the Fourier expansion of the generalized damping function.

The second-mode correction amplitude $q_{21}$ in (39) is given by (38). For a pipe which is perfect (cf. Sect. 2.5) except for a nonzero mass flow $(\alpha \nu \neq 0)$, and (as with flowmeters) is driven at a nodal point for the second vibration mode $\left(\varphi_{2}\left(x_{p}\right)=0\right)$, the only nonzero term is the one with $\hat{c}_{21}$; for this case, by (15) and (12), $q_{21}=2 C_{21} a \alpha v \omega_{1}\left(\omega_{2}^{2}-\omega_{1}^{2}\right)^{-1} \sin (\Omega t-\psi)$, which describes a second-mode component oscillating $90^{\circ}$ out of phase with the primarily excited first mode, but at the same frequency, with an amplitude proportional to the mass flow $\alpha v$; this is the design case for a Coriolis flowmeter. By (39) the resulting pipe motion $u(x, t)$ is then a traveling 
wave, i.e. the nodes of the vibration pattern move in time; this corresponds to a phase shift in the zero crossing times for two different points located along the pipe, which can be measured and related to the mass flow. However, as appears from (38) a nonzero value of $\beta \int_{0}^{1} \varphi_{2} \xi_{n} \mathrm{~d} x$ (i.e. nonlinear, asymmetric damping, cf. (32)) can also produce a second-mode oscillation at frequency $\Omega$ and $90^{\circ}$ degrees out of phase with the driven mode, and thus traveling waves that could be erroneously related to mass flow. The same applies if $c_{21} \neq 0$, as could occur with even linear damping varying nonuniformly along the pipe-axis; this would lead to a change of $\hat{c}_{21}$ which is unrelated to mass flow (cf. (15)).

To investigate more closely the effects of pipe nonuniformity and generalized nonlinear damping, we next calculate predictions of the phase shift between two specific points located along the pipe; this is what is actually measured in Coriolis flowmeter applications.

\section{Phase shift with mid-pipe sharply resonant excitation}

\subsection{Resonant response amplitude at measurement locations}

With Coriolis flowmeters, pipe motions are typically measured by a pair of magnetic pickup coils situated at $x=x_{1}$ and $x=x_{2}$ symmetrically an axial distance $\Delta x$ from the pipe middle:

$$
\left.x_{1,2}=\frac{1}{2} \mp \Delta x, \Delta x \in\right] 0 ; \frac{1}{2}[\text {. }
$$

The pickup signals are narrow-band filtered and analyzed to ensure the measured phase shift between $x_{1}$ and $x_{2}$ is only for vibrations at the excitation frequency $\Omega$. The pipe excitation is applied at $x_{p}=\frac{1}{2}$, so that $\varphi_{2}\left(x_{p}\right)=0$, cf. (7), and is controlled by velocity-feedback to be sharply resonant with the damped primary resonance frequency, so that and $\Omega=\hat{\omega}_{1}^{*}$ and $\hat{a}=\hat{a}^{*}$, cf. Fig. 2 and (45)-(46). Under these conditions, and according to (39) with (37)-(38), recalling that only vibration components at frequency $\Omega$ passes the filter, the pipe motions measured at $x=x_{k}$ becomes:

$$
\begin{aligned}
u\left(x_{k}, t\right) & =\hat{a}\left[A\left(x_{k}\right) \sin \left(\Omega T_{0}-\hat{\psi}\right)+B\left(x_{k}\right) \cos \left(\Omega T_{0}-\hat{\psi}\right)\right]+O\left(\varepsilon^{2}\right) \\
& =\hat{a} \sqrt{A\left(x_{k}\right)^{2}+B\left(x_{k}\right)^{2}} \cos \left(\Omega T_{0}-\hat{\psi}+\Psi\left(x_{k}\right)\right)+O\left(\varepsilon^{2}\right),
\end{aligned}
$$

where

$$
\begin{gathered}
A(x)=\varepsilon \frac{\omega_{1} \hat{c}_{21}}{\omega_{2}^{2}-\omega_{1}^{2}} \varphi_{2}(x), \quad B(x)=\varphi_{1}(x)+\varepsilon \frac{\omega_{1}^{2} m_{21}-K_{21}}{\omega_{2}^{2}-\omega_{1}^{2}} \varphi_{2}(x), \\
\Psi(x)=\arctan (A(x) / B(x)),
\end{gathered}
$$

and where for $A(x)$ a term $2 \beta \int_{0}^{1} \varphi_{2} \xi_{1} \mathrm{~d} x$ has cancelled, being the integral of a product of an antisymmetric and a symmetric function, cf. (7) and the comment below (32). To order $\varepsilon$ the pipe response amplitude $\hat{u}\left(x_{k}\right)$ at pipe measurement point $x_{k}$ is then, by Taylor-expanding the factor multiplying the dominating harmonic term in (48) and inserting (49):

$$
\hat{u}\left(x_{k}\right)=\hat{a}\left(\varphi_{1}\left(x_{k}\right)+\varepsilon \frac{\omega_{1}^{2} m_{21}-K_{21}}{\omega_{2}^{2}-\omega_{1}^{2}} \varphi_{2}\left(x_{k}\right)\right)+O\left(\varepsilon^{2}\right)
$$




\subsection{Phase shift between measurement locations}

Defining the phase shift between the measurement points $x_{1}$ and $x_{2}$ as

$$
\Delta \Psi=\Psi\left(x_{1}\right)-\Psi\left(x_{2}\right),
$$

and utilizing that (by (47) and (7)) $\varphi_{1}\left(x_{1}\right)=\varphi_{1}\left(x_{2}\right)$ and $\varphi_{2}\left(x_{1}\right)=-\varphi_{2}\left(x_{2}\right)$, gives, upon Taylorexpanding for small $\varepsilon$, and substituting (15) with (11) and (20) with (11)-(12) for $\hat{c}_{21}$ :

$$
\Delta \Psi=\varepsilon \frac{2 \omega_{1} \varphi_{2}\left(x_{1}\right)}{\left(\omega_{2}^{2}-\omega_{1}^{2}\right) \varphi_{1}\left(x_{1}\right)}\left(2 \alpha \nu \int_{0}^{1} \varphi_{2} \varphi_{1}^{\prime} \mathrm{d} x+c_{12}\right)+O\left(\varepsilon^{3}\right)
$$

where, by (12):

$$
\begin{aligned}
c_{12} & =\int_{0}^{1} c_{u}(x) \varphi_{1} \varphi_{2} \mathrm{~d} x+\int_{0}^{1} c_{\theta}(x) \varphi_{1}^{\prime} \varphi_{2}^{\prime} \mathrm{d} x \\
& =\frac{1}{2} \int_{0}^{1}\left(c_{u}(x)-c_{u}(1-x)\right) \varphi_{1} \varphi_{2} \mathrm{~d} x+\frac{1}{2} \int_{0}^{1}\left(c_{\theta}(x)-c_{\theta}(1-x)\right) \varphi_{1}^{\prime} \varphi_{2}^{\prime} \mathrm{d} x,
\end{aligned}
$$

where the last equality follows from splitting $c_{u}$ and $c_{\theta}$ into symmetric and antisymmetric parts and noting the symmetry properties of the mode shapes (for hinged-hinged or clampedclamped pipes $\varphi_{1} \varphi_{2}$ and $\varphi_{1}^{\prime} \varphi_{2}^{\prime}$ are both antisymmetric on $\left.x \in[0,1]\right)$.

The analytical prediction (52) for the phase shift is accurate to order $\varepsilon^{2}$ (the $\varepsilon^{2}$-terms cancel identically); it can readily be rearranged into a form more directly useful for applications:

$$
\Delta \Psi=\varepsilon\left(s_{1} \alpha \nu+\Delta \Psi_{0}\right)+O\left(\varepsilon^{3}\right)
$$

where $s 1$ is the linear meter sensitivity, i.e. the factor of proportionality between mass flow $\alpha v$ and phase shift $\Delta \Psi$ :

$$
S_{1}=2 \Gamma \int_{0}^{1} \varphi_{2} \varphi_{1}^{\prime} \mathrm{d} x, \quad \Gamma=\frac{2 \omega_{1}}{\omega_{2}^{2}-\omega_{1}^{2}} \frac{\varphi_{2}\left(x_{1}\right)}{\varphi_{1}\left(x_{1}\right)},
$$

and $\Delta \Psi_{0}$ is the zero shift, i.e. the phase shift present when there is no fluid flow $(\alpha \nu=0)$ :

$$
\Delta \Psi_{0}=c_{12} \Gamma \text {. }
$$

Note again that $\varepsilon$ only serves to bookmark the magnitude order of small terms; in actual numerical calculation it is set to unity. From (54)-(56) some conclusions relevant for flowmetering applications readily follow:

To order $\varepsilon^{2}$, the meter sensitivity $s$ is predicted to:

- grow with the nearness of the perfect-pipe natural frequencies $\omega_{1}$ and $\omega_{2}$ for the two vibration modes involved, in the same proportion as reported in other studies $[1,45]$,

- be independent on the vibration amplitude $\hat{a}$, and

- be independent on all imperfections included in this study, i.e. small $(O(\varepsilon))$ linear and nonlinear damping, mass and stiffness nonuniformity, and additional transverse stiffness (by pre-tension, midplane stretching / symmetric stiffness nonlinearity, or asymmetric nonlinearity).

Also, according to (56) a zero shift $\Psi_{0 \neq 0}$ may result if $c_{12} \neq 0$. According to (53) this generally occurs if $c_{u}(x) \neq c_{u}(1-x)$ or $c \theta(x) \neq c \theta(1-x)$ i.e. if the damping distributions are not symmetric w.r.t. $x=\frac{1}{2}$ on $x \in[0,1]$. In practice zero phase shifts of (also) this kind are routinely removed 
during the initial flowmeter calibration. However, subsequent changes of system damping under operation (e.g. due to temperature, wear, lubrication, vibration level, or multi-phase flow [21]) could lead to phase shifts that would erroneously be related to mass flow.

It is important to note that these conclusions only holds under the assumptions that all imperfections considered are small. Thus the rather general expressions (54)-(56) does not imply that the phase shift, the meter sensitivity, and the zero shift are independent of all imperfections considered in this study other than mass flow and asymmetric damping. What can be inferred is only that if the imperfections considered are of magnitude order $O(\varepsilon), \varepsilon \ll 1$, then the effect of asymmetric damping on phase shift is of the same order of magnitude as the mass flow, i.e. $O(\varepsilon)$, and introduces a zero shift that could be mistaken for mass flow, while the effect of all other imperfections considered are at least two orders of magnitude, smaller, i.e. $O\left(\varepsilon^{3}\right)$. As for imperfection in the form of added nonuniform mass, a similarly very weak dependency on phase shift was reported in [46].

Indeed the phase shift does depend on several of the imperfections considered here, if just large enough. Added mass, for example, changes the separation between the two lowest natural frequencies and the mode shapes, and thus affects meter sensitivity. This is evident e.g. from Fig. 4 in [10], showing a clear effect of added mass on phase shift; however in that example the total mass amounts to up to $30 \%$ of the pipe mass, and thus is not a "small imperfection" in the sense considered in the present paper (where the corresponding terms in the equation of motion should be much smaller than the dominating terms). In [10] the added mass is not assumed small, and thus the resulting analytical expressions are more accurate than (54)-(56), but also significantly more complicated and less readily interpretable. (See e.g. [23] for a further discussion of added mass effects.)

\subsection{Phase shift with hinged supports}

The above conclusions are valid for both sets of boundary conditions considered (hingedhinged, clamped-clamped), as long as the pickups are symmetrically positioned lengthwise, and the excitation applied at the pipe center, $x_{p}=\frac{1}{2}$. For hinged supports (52) gives, on inserting (6)-(7) and (47):

$$
\Delta \Psi=\varepsilon \frac{64}{45 \pi^{2}} \sin (\pi \Delta x)\left(\alpha v+\frac{3}{16} c_{12}\right)+O\left(\varepsilon^{3}\right),
$$

where, by (53):

$$
\begin{aligned}
c_{12}= & \int_{0}^{1}\left(c_{u}(x)-c_{u}(1-x)\right) \sin (\pi x) \sin (2 \pi x) \mathrm{d} x \\
& +2 \pi^{2} \int_{0}^{1}\left(c_{\theta}(x)-c_{\theta}(1-x)\right) \cos (\pi x) \cos (2 \pi x) \mathrm{d} x .
\end{aligned}
$$

This is identical to what was found in [1] for the case of ideally hinged supports (i.e. with $\kappa_{1}=$ 0 in [1], Eq. (42)-(43)). According to (57) the maximum linear meter sensitivity is obtained for $\Delta x \rightarrow \frac{1}{2}$, i.e. with measurement pickups placed as close as possible to the supports, however, in practice pickups are located so as to obtain the strongest signal from the (weakly) flow-excited second vibration mode, i.e. at the antinodes of $\varphi_{2}$, corresponding to $\Delta x=\frac{1}{4}$ for hinged supports. 


\section{Numerical validation of analytical predictions}

The approximate analytical predictions of phase shift (52) and (57) can be tested by comparing with numerical results obtained with a minimum of approximating assumptions, using a Galerkin expansion a with sufficiently high number of modes to discretize (1). For the linear problem $(\mu=\gamma=\beta=0)$ numerical solutions for the pipe motion can be calculated by inserting the known solution form and solving the resulting set of linear algebraic equations for the modal amplitudes; phase shifts can then be calculated without analyzing response time series. For the nonlinear problem, by contrast, numerical simulation of the ordinary nonlinear differential equations for the modal amplitudes is required.

\subsection{Main approximations and solution procedure}

The partial differential equation (1) is discretized by the standard Galerkin expansion (9), using mode shapes (7) of the corresponding unperturbed problem. The resulting system of $N$ coupled second order nonlinear differential equations (10) can be written in the form:

$$
\mathbf{M} \ddot{\mathbf{q}}+\mathbf{D}(v) \dot{\mathbf{q}}+\mathbf{K}(v) \mathbf{q}+\mathbf{g}(\mathbf{q}, \dot{\mathbf{q}})=\mathbf{f} \cos (\Omega t) .
$$

where $\mathbf{q}=\mathbf{q}(t)$ holds the modal amplitudes $q_{j}(t), j=1, \ldots, N$, and the elements of the mass matrix $\mathbf{M}$, the damping and stiffness matrices $\mathbf{D}(v)$ and $\mathbf{K}(v)$ (both fluid-velocity dependent), the essentially nonlinear forcing vector $\mathbf{g}(\mathbf{q}, \dot{\mathbf{q}})$, and the modal forcing amplitude vector $\mathbf{f}$ are:

$$
\begin{gathered}
\mathbf{M}_{i j}=\delta_{i j}+\varepsilon m_{i j}, \quad \mathbf{D}_{i j}(v)=\varepsilon \hat{c}_{i j}, \quad \mathbf{K}_{i j}(v)=\delta_{i j} \omega_{j}^{2}+\varepsilon K_{i j}, \\
\mathbf{g}_{i}(\mathbf{q}, \dot{\mathbf{q}})=\varepsilon\left[\gamma \sum_{j, k=1}^{N} H_{i j k} q_{j} q_{k}+\frac{1}{2} \mu^{2} \sum_{r, s=1}^{N} B_{r s} q_{r} q_{s} \sum_{j=1}^{N} B_{i j} q_{j}+\beta \int_{0}^{1} \varphi_{i} f\left(\sum_{j=1}^{N} \dot{q}_{j} \varphi_{j}\right) \mathrm{d} x\right], \\
\mathbf{f}_{i}=\varepsilon p \varphi_{i}\left(x_{p}\right), \quad i, j=1,2, \ldots, N,
\end{gathered}
$$

where all system constants are already defined (cf. (5), (6), (11), (12), (15)), and $\varepsilon$ still serves only to bookmark terms assumed to be small (in actual numerical calculations $\varepsilon=1$ ).

For $N=2$ this system reduces to the two-mode approximation (14), which allowed the simple analytical approximate expression (52) for the phase shift to be set up, though at the cost of reduced accuracy due to excluding vibration modes higher than the second. Using numerical simulation of (59)-(60) for sufficiently high $N$ allows the accuracy of (52) to be tested.

\subsubsection{Determining phase shift in the linear case}

For the linear case $\gamma=\mu=\beta=0$, and thus $\mathbf{g = 0}$. Then (59) can be solved exactly for $\mathbf{q}(t)$, and the resulting phase shift calculated in a straightforward manner, as described for a similar case in [1]. For this the pipe is assumed to be excited resonantly in its fundamental symmetric mode. The resonance frequency of this mode changes with fluid flow and other imperfections considered; it can be calculated by letting $\mathbf{f}=\mathbf{g}=\mathbf{0}$ in (59), inserting a time harmonic solution $\mathbf{q}(t)=\varphi^{*} \mathrm{e}^{\lambda t}$, and solving the resulting algebraic eigenvalue problem numerically for the fundamental eigenvalue $\lambda=\lambda_{1}$. Generally with underdamped systems, and even with nonzero flow speed $\nu \neq 0$ or if $\mathbf{D}$ is not proportional to $\mathbf{K}$ or $\mathbf{M}$, the eigenvalues $\lambda=\lambda_{j}$ come as complex conjugate pairs, with the imaginary part $\operatorname{Im}\left(\lambda_{j}\right)=\omega_{j}^{*}$ defining the $j$ 'th damped natural frequency, and the real part defining the damping ratio $\zeta_{j}=-\operatorname{Re}\left(\lambda_{j}\right) /|\lambda|$ of mode $j$ [47]. Thus, as the excitation frequency for resonant excitation of the fundamental mode we take $\Omega=\omega_{1}{ }^{*}$ ( $\approx \hat{\omega}_{1}=\hat{\omega}_{1}^{*}$ in the absence of midplane stretching nonlinearity, $\mu=0$, cf. Fig. 2 ). 
To solve (59) for the harmonically forced linear case $(\mathbf{f} \neq \mathbf{0}, \mathbf{g}=\mathbf{0})$ we insert a time-harmonic solution form for the stationary part of the response:

$$
\mathbf{q}(t)=\mathbf{a} \sin (\Omega t)+\mathbf{b} \cos (\Omega t),
$$

and separate the in-phase $(\cos (\Omega t))$ and out-of-phase $(\sin (\Omega t))$ terms to give:

$$
\left[\begin{array}{cc}
\mathbf{K}-\Omega^{2} \mathbf{M} & -\Omega \mathbf{D} \\
\Omega \mathbf{D} & \mathbf{K}-\Omega^{2} \mathbf{M}
\end{array}\right]\left\{\begin{array}{l}
\mathbf{a} \\
\mathbf{b}
\end{array}\right\}=\left\{\begin{array}{l}
\mathbf{0} \\
\mathbf{f}
\end{array}\right\},
$$

which can be solved for the vectors $\mathbf{a}$ and $\mathbf{b}$ to give the corresponding $\mathbf{q}(\mathrm{t})$ by (61) for any excitation frequency $\Omega$, including the particular 1 st-mode resonance frequency $\omega_{1}{ }^{*}$ of interest here.

Substituting $\Omega=\omega_{1}^{*}$ into (61)-(62), and solving (62) for the corresponding resonant values of $\mathbf{a}=\left\{a_{1} \cdots a_{N}\right\}^{T}$ and $\mathbf{b}=\left\{b_{1} \cdots b_{N}\right\}^{T}$, the value of $\mathbf{q}$ resulting from (61) can be substituted into (9) to give, upon rewriting from sine-cosine to amplitude-phase form:

$$
u(x, t)=C(x) \sin \left(\omega_{1}^{*} t-\Psi(x)\right)
$$

where the amplitude $C$ and the phase $\Psi$ generally vary along the pipe axis $x$ :

$$
\begin{gathered}
C(x)=\sqrt{\left(\sum_{j=1}^{N} a_{j} \varphi_{j}(x)\right)^{2}+\left(\sum_{j=1}^{N} b_{j} \varphi_{j}(x)\right)^{2}}, \\
\Psi(x)=-\arctan \left(\sum_{j=1}^{N} b_{j} \varphi_{j}(x) / \sum_{j=1}^{N} a_{j} \varphi_{j}(x)\right),
\end{gathered}
$$

The phase shift $\Delta \Psi$ between any two pipe points $x_{1}$ and $x_{2}$ can then be calculated by inserting (65) into (51). For two symmetrically situated points $x_{1,2}=\frac{1}{2} \mp \Delta x$ (cf. (47)) this gives:

$$
\Delta \Psi=\Psi\left(\frac{1}{2}-\Delta x\right)-\Psi\left(\frac{1}{2}+\Delta x\right),
$$

the numerical result of which can then be compared to the corresponding phase shift predicted by the simple analytical approximations (52) or (57).

Eq. (66) with (65) will be referred to as the numerical solution, since it relies on numerical solution of the set of linear algebraic equations (62); it was calculated using MATLAB. The convergence of numerically calculated phase shifts $\Delta \Psi$ with increased number of included modes $N$ was tested in each case reported below, with final values of $N$ chosen large enough to ensure no significant changes in results by halving or doubling $N$.

The numerical solution can be expected to offer good accuracy as $N$ is increased, converging towards the exact solution as $N \rightarrow \infty$. It does not require imperfections to be small, but on the other hand provides very little insight into how imperfections affect phase shift. Thus the key role of numerical solution is here to test the quality of the simple, more approximate analytical solutions. For parameter ranges where the analytical expressions can be validated, these are considered much more useful than numerical solutions for practical innovation, design, and troubleshooting.

\subsubsection{Determining phase shift and response amplitude in the nonlinear case}

For the nonlinear case at least one of $\left(\gamma, \beta, \mu^{2}\right)$ is nonzero, so that $\mathbf{g} \neq \mathbf{0}$ in (59)-(60). Then this set of nonlinear ODEs must be solved for $\mathbf{q}(t)$ for specific parameters by numerical integration, 
with the excitation frequency equal to the peak resonant frequency, $\Omega=\hat{\omega}_{1}^{*}$ (cf. Fig. 2), and the corresponding pipe deformations $u\left(\frac{1}{2} \pm \Delta x, t\right)$ calculated by (9). The procedure follows [18] in first rewriting (59) into standard (implicit) first-order form:

$$
\tilde{\mathbf{M}} \dot{\mathbf{y}}=\mathbf{G}(t, \mathbf{y}),
$$

where $\mathbf{y}=\mathbf{y}(t)=\left\{\begin{array}{ll}\mathbf{q}^{T} & \dot{\mathbf{q}}^{T}\end{array}\right\}^{T} \in R^{2 N}$, and:

$$
\tilde{\mathbf{M}}=\left[\begin{array}{cc}
\mathbf{I} & \mathbf{0} \\
\mathbf{0} & \mathbf{M}
\end{array}\right], \quad \mathbf{G}(t, \mathbf{y})=\left[\begin{array}{cc}
\mathbf{0} & \mathbf{I} \\
-\mathbf{K} & -\mathbf{D}
\end{array}\right] \mathbf{y}-\left\{\begin{array}{c}
\mathbf{0} \\
\mathbf{g}(\mathbf{y})
\end{array}\right\}+\left\{\begin{array}{l}
\mathbf{0} \\
\mathbf{f}
\end{array}\right\} \cos (\Omega t) .
$$

with $\mathbf{M}, \mathbf{D}, \mathbf{K}, \mathbf{g}$, and $\mathbf{f}$ given by (60). Equation (67) with (68) was solved for $\mathbf{y}=\mathbf{y}(t)$ using a MATLAB standard solver for stiff systems (ODE23TB, and crosschecking with other solvers),

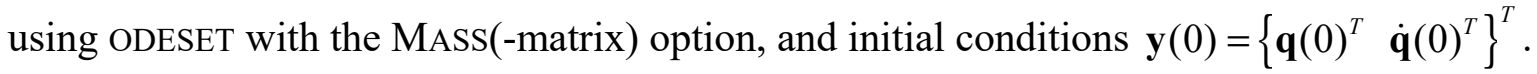

When during simulation a stationary state has settled, the time shift $\Delta t$ between the instances of zero velocity crossings of $u$ at the two pipe points $x_{1,2}=\frac{1}{2} \mp \Delta x$ can be determined numerically using (9), and the corresponding phase shift $\Delta \Psi=\Omega \Delta t$ calculated. With numerical solutions that are time-sampled uniformly at high enough frequency, and zero crossings determined by linear interpolation between sampled points, the time shift $\Delta t$ can be determined accurately enough to reveal even the very small phase shifts $\Delta \Psi$ relevant for flowmeter applications.

\subsubsection{Numerical issues with nonlinear response simulation}

a) Convergence tolerance: A proper choice of MATLAB's absolute tolerance parameter (ABSTOL) turned out to be more than usually critical for acceptable numerical solution accuracy: Whereas the choice of the relative tolerance parameter (RELTOL) is not critical, MATLAB's default value of $10^{-6}$ for ABSTOL in most cases implies highly inaccurate numerical results for the phase shift $\Delta \Psi$. This is because the phase shift depends mostly on the nondimensional modal amplitudes $q_{1}$ and $q_{2}$ of the first and second vibration mode (cf. (9)), where for realistic flowmeter parameters $q_{1}$ is very small, e.g. $O\left(10^{-6}\right)$, and $q_{2}$ maybe an order of magnitude smaller, and the higher modes yet smaller. With MATLAB's ODE-solver considering all solution components smaller than ABSTOL effectively zero or "unimportant", ABSTOL needs to be set small enough that the smallest component relevant for the solution (typically that means the amplitude of the highest mode included) will be determined to full accuracy, i.e. with tolerance RELTOL. (Or alternatively, the modal amplitudes $q_{j}$ could be rescaled to be all of order unity.) For all results presented below parameters were set to $($ ReLToL,ABSTOL $)=\left(10^{-8}, 10^{-10}\right)$, giving acceptable accuracy and computation time.

b) Initial conditions: In some cases choosing these is not trivial, since with nonlinear systems the stationary state may depend on initial conditions. With weak cubic nonlinearities and mono-frequency harmonic excitation as assumed, the present system will have at most two stable stationary states at the drive frequency $\Omega=\hat{\omega}_{1}^{*}$ (cf. Fig. 2). However, with a real Coriolis flowmeter, feedback control will work so as to keep the response close to the backbone of the frequency response (dash-dotted in Fig. 2), since this gives the relation between oscillation frequency and amplitude for an undamped and unforced system, and this is effectively what the feedback control does: delivers a small amount of energy at the natural frequency corresponding to a given amplitude, which exactly balances the energy dissipated by damping, so that the system is held at a constant frequency and amplitude. This means that the solution for 
the feedback-controlled system will be at $\left(\hat{\omega}_{1}^{*}, \hat{a}^{*}\right)$ in Fig. 2 , i.e. at the peak, and not on the lower branch. Thus we use, for the excitation frequency $\Omega$, the analytically predicted resonance frequency $\hat{\omega}_{1}^{*}$ given by (46). But for cases with significantly bent response peak (i.e. with significant stiffness nonlinearity), a numerical procedure was employed corresponding to positive velocity-feedback (i.e. negative damping), ensuring the solution to be at the top of the response peak and not on the lower branch.

c) Achieving stationarity: The inherently very low damping in Coriolis flowmeter pipes, implies similarly long simulation times needed for transients to decay and stationarity to settle. To indicate the time scale, for a first-mode damping ratio $\zeta_{1} \approx 0.01 \%$ (a typical value for Coriolis flowmeters, cf. Sect. 5.2 below) the number of oscillation cycles $N_{R}$ needed to reach $R=$ $99.9 \%$ of the stationary amplitude is $n_{R, 1} \approx-\ln (1-R) /\left(2 \pi \zeta_{1}\right) \approx 11,000$ cycles of first-mode oscillations. To drastically cut down on the simulation time needed to attain stationarity, the already known linear stationary solution (61), giving $\mathbf{q}\left(t_{0}\right)$ at some arbitrary time $t_{0}$, was used as initial conditions $\mathbf{q}(0)$ for the nonlinear system (67). To further cut down on simulation time, these initial conditions were multiplied by $\hat{a}^{*} / \hat{a}\left(\hat{\omega}_{1}\right)$, i.e. by the theoretically predicted ratio between nonlinear and linear base oscillation amplitude (cf. (42), (43), (45)). By running a few simulation instances at very long times corresponding to at least $n_{R, 1}$ cycles, it was verified that stationarity was actually reached.

d) Coping with long computation times: With e.g. $N=16$ modes the natural frequency of the highest mode of the hinged-hinged pipe is $16^{2}=256$ times that of the fundamental mode, making the system computationally stiff, and requiring fine time discretization. When additionally the damping is extremely low, requiring many oscillation cycles simulation to reach stationarity (cf. preceding paragraph), and nonlinearity is also involved, then computation time for a standard PC running MATLAB becomes of the order of days to simulate a useful time series for just a single set of parameter values. This problem is reduced drastically when increasing the damping coefficient away from the extremely low value encountered with real Coriolis flow meters. Therefore, for the nonlinear cases reported below, the damping is artificially increased by a factor 100 , using a base value of $c_{u 0}=0.2$ (corresponding to $\zeta_{1}=1.0 \%$ and $\zeta_{2}=0.25$ $\%$, i.e. still very weak damping), and at the same time increasing also the forcing amplitude $p$ by the same factor, so as to maintain the same level of stationary vibration amplitude (about $7 \times 10^{-5}$; see below) in all cases. This cuts down the time to reach stationarity during simulation by about the same factor, while supposedly not affecting the main conclusion drawn from the validation procedure. The latter is due to the way the artificial damping is introduced, as a simple increase in the spatially uniform part of the system damping, which is already known $[1,20,48]$ not to affect the spatial phase shift of interest here (this damping affects only the phase temporal shift between input forcing and output response). Furthermore, for some of the numerical simulations with nonlinearity, the number of included modes was reduced to $N=8$, as indicated in the figure captions, checking for a few cases that doubling the number of modes would not visibly change the graphs displayed. Alternative ways to cut down simulation time could involve numerically more efficient software, e.g. using Fortran coding, or replacing brute force simulation with solvers searching for periodic solutions (e.g. MATLAB's BVP solver BVP5C).

e) Calculating stationary vibration amplitude from time series data with weak harmonic distortion: With numerical simulation the response amplitude $\hat{u}_{1}$ at pipe measurement point $x_{1}$ is calculated simply as half the peak-to-peak amplitude in the stationary time series $u\left(x_{1}, t\right)$, ignoring the very small content of harmonic distortion. The value of $\hat{u}_{1}$ so computed can be compared to the analytical prediction given by (50). The distorting higher harmonics could be 
filtered away, if significant; this is done anyway with a real Coriolis flow meter, where only the signal components at the drive frequency $\Omega$ are further processed.

\subsection{Validation cases}

The simple first-order approximate analytical prediction (57)-(58) for phase shifts of the hinged-hinged beam is here validated against numerical solutions, at the same time also illustrating the effect of the considered pipe imperfections on the phase shift. After defining baseline system parameter values, sects. 5.2.2-5 consider the effects of imperfections corresponding to, respectively, nonuniform linear damping, nonuniform mass and stiffness, nonlinear damping, and nonlinear stiffness.

\subsubsection{Baseline system parameters}

The baseline parameters are for a perfect pipe, except that uniform transverse damping is included in all cases, i.e. $\Delta m(x)=\Delta e(x)=c_{\theta}(x)=\mu=\eta=\gamma=\beta=0$ (cf. Sect. 2.5), but $c_{u}(x) \neq 0$. The effects of imperfections are then considered separately. For the uniform transverse damping $c_{u}(x)=c_{u 0}=0.002$, unless otherwise stated in figure captions; this implies a quality factor $Q_{1}$ $=\omega_{1} / c_{u} \approx 5000$ for the drive mode, or a damping ratio of $\zeta_{1}=\left(2 Q_{1}\right)^{-1} \approx 0.010 \%$ (and $\zeta_{2}=0.0025$ $\%$ ) corresponding to the value for a particular industrial Coriolis flowmeter. For the mass ratio we use $\alpha=0.3$, and consider a mass flow range $\alpha v \in[0,0.1]$; this roughly corresponds to a specific industrial Coriolis flowmeter measuring water flow from zero to full nominal flow rate. For optimal flowmeter sensitivity the measurement sensors are located at the antinodes of the second vibration mode, i.e. $x_{1}=\frac{1}{4}, x_{2}=\frac{3}{4}, \Delta x=\frac{1}{4}$, while input forcing is provided at the antinode of the first mode, i.e. $x_{p}=\frac{1}{2}$, with amplitude $p=10^{-6}$ (unless otherwise stated in figure captions); this exemplifies, for the level of damping used, a flowmeter pipe vibrating at maximum normalized resonant amplitude of the order $7 \times 10^{-5}$, e.g. a $200 \mathrm{~mm}$ long pipe vibrating at about $14 \mu \mathrm{m}$ amplitude.

\subsubsection{Effect of nonuniform linear damping}

As an illustrative example we consider the damping distribution to be basically uniform, but with an additional nonuniformity localized at $x=x_{c} \in\left\{x_{c u}, x_{c \theta}\right\}$, , i.e.:

$$
\begin{aligned}
& c_{u}(x)=c_{u 0}+c_{u 1} \delta\left(x-x_{c u}\right), \\
& c_{\theta}(x)=c_{\theta 0}+c_{\theta 1} \delta\left(x-x_{c \theta}\right),
\end{aligned}
$$

where $c_{u 0}$ and $c_{\theta 0}$ are positive constants for uniform transverse and rotational damping, respectively, and $c_{u 1}$ and $c_{\theta 1}$ the corresponding constants for a localized change (positive or negative) in damping at $x=x_{c u}$ and $x=x_{c \theta}$. The phase shift $\Delta \Psi$ is then given by (57), where $c_{12}$ is calculated from (58) with (69)-(70) to give:

$$
c_{12}=2 c_{u 1} \sin \left(\pi x_{c}\right) \sin \left(2 \pi x_{c}\right)+4 \pi^{2} c_{\theta 1} \cos \left(\pi x_{c}\right) \cos \left(2 \pi x_{c}\right),
$$

which does not depend on the uniform damping constants $c_{u 0}$ and $c_{\theta 0}$. Thus to order $\varepsilon^{2}$ the phase shift is predicted to be unaffected by small $(O(\varepsilon))$ uniformly distributed linear viscous damping. Also, transverse damping localized at the nodes of the first or second vibration mode $\left(x_{c}=\frac{1}{2}\right)$ has no effect on the phase shift (the $c_{u 1}$-term in (71) vanishes); similarly, rotational damping localized at the antinodes of the first or second mode $\left(x_{c} \in\left\{\frac{1}{4}, \frac{1}{2}, \frac{3}{4}\right\}\right)$ has no effect on the phase shift (the $c_{\theta 1}$-term vanishes). 
Fig. 3(a) shows the effect of localized (i.e. nonuniform) transverse damping $c_{u 1}$ (cf. (69)) on the phase shift $\Delta \Psi$ for varying mass flow $\alpha v$, with details as given in the caption, and with the uniform part of the damping $c_{u 0}=0.002$ (cf. intro to Sect. 5.2). Solid lines represent results obtained by the analytical approximation (57) with (71); they agree very well with results of numerical solution (65)-(66) of the original model equations (1)-(3), deviating less than 0.6 $\%$ even for the largest nonuniformity, $c_{u 1}= \pm 0.2$, which is two orders of magnitude higher than the uniform part of the damping. The number of modes used in the Galerkin expansion (9) were $N=16$, chosen so as to ensure convergence of $\Delta \Psi$ to typically six (in worst cases three) significant digits. However, the results appear to be rather insensitive to all $N \geq 2$, reflecting the dominating influence of the lowest two modes. This also explains the high accuracy of the simple analytical prediction, which include just these modes.

Fig. 3(b) illustrates the effect of different levels of pure uniform damping $c_{u 0}$ (with $c_{u 1}=0$ ) on the phase shift $\Delta \Psi$. The values used for $c_{u}$ spans four orders of magnitudes, with corresponding damping ratios in the range $0.01-10 \%$, i.e. from realistic to unrealistically high, but in all cases with good agreement between analytical predictions and numerical solutions, and demonstrating independency of the phase shift to uniformly distributed linear viscous damping (all lines coinciding and symbols overlaid).

As for rotational damping (cf. (70)), corresponding graphs for the effects of the nonuniform part $c_{\theta 1}$ and the uniform part $c_{\theta 0}$ are not given here, since they are closely similar to Fig. 3(a) and (b) for transverse damping, that is: The nonuniform part introduces zero shifts (i.e. the lines translate upwards with increasing nonuniformity), the uniform part has no effect on phase shift (all lines coinciding), and the agreement of the analytical prediction (57) with (71) to numerical solutions is very good, even up to unrealistically high values of the damping constants.

The prediction that asymmetrically distributed damping introduces a zero shift, while symmetrically (including uniformly) distributed damping has no significant effect, agrees with experimental findings involving artificially induced damping asymmetries [20,49].
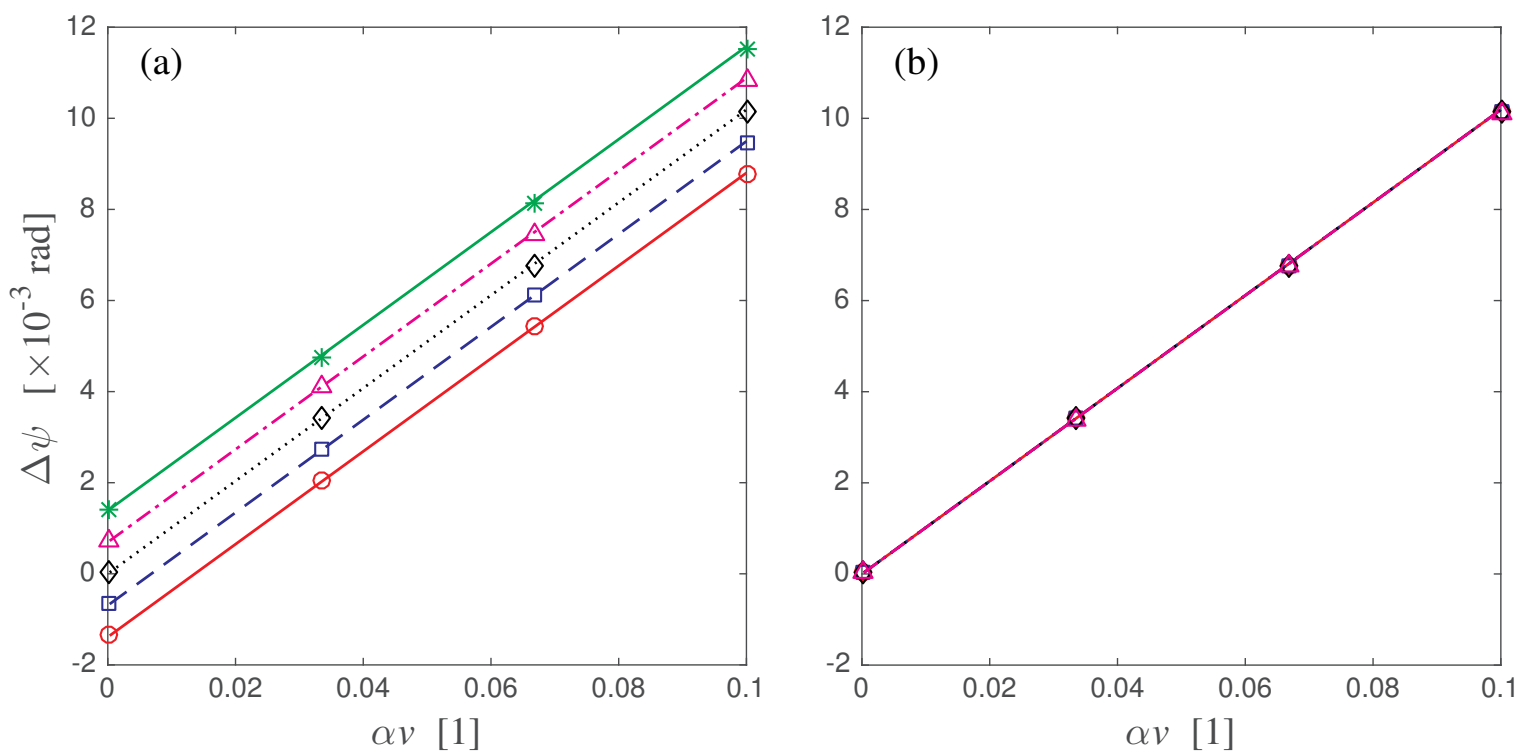

Fig. 3 (a) Effect of localized (i.e. nonuniform) transverse damping $c_{u 1}$ (cf. (69)) on phase shift $\Delta \Psi$ for varying mass flow $\alpha v$, as obtained by the analytical approximation (57) with (71) (lines), and by the numerical solution (65)-(66) to (1)-(3) using $N=16$ modes (symbol markers). From bottom to top the lines show $\Delta \Psi$ for different levels of nonuniform damping $c_{u 1}=\{-0.2,-0.1,0.0,0.1,0.2\}$ at location $x_{c u}=0.1$, with $c_{u 0}=0.002$ in all cases. (b) Effect of different levels $c_{u 0}=0.002 \times\{1,10,100,1000\}$ of pure uniform transverse damping $\left(c_{u 1}=0\right)$ on $\Delta \Psi$, again with lines (symbols) showing results of numerical solution (analytical approximation) 


\subsubsection{Effect of nonuniform mass and stiffness}

As an example of mass and stiffness nonuniformity we consider the following axial distribution of, respectively, additional (fluid empty) pipe mass $\Delta m$, additional bending stiffness $\Delta e$, transverse stiffness $k_{u}$, and rotational stiffness $k_{\theta}$ :

$$
\begin{aligned}
\Delta m(x) & =m_{1} \delta\left(x-x_{m}\right), \\
\Delta e(x) & =e_{1} \delta\left(x-x_{e}\right), \\
k_{u}(x) & =k_{u 1} \delta\left(x-x_{k u}\right), \\
k_{\theta}(x) & =k_{\theta 1} \delta\left(x-x_{k \theta}\right),
\end{aligned}
$$

where $m_{1}, e_{1}, k_{u 1}, k_{\theta 1}$ represent concentrated added (or removed) mass and stiffness at corresponding locations $x \in\left\{x_{m}, x_{e}, x_{k u}, x_{k q}\right\}$.

Fig. 4(a)-(d) displays the relation between phase shift $\Delta \Psi$ and mass flow $\alpha v$ for each of the four nonuniformities separately, with solid lines representing the analytical approximation (57) with (71) and (72), symbols marking numerical solutions (65)-(66) of the original model equations (1)-(3), and parameter values as given in the caption. Numerical solutions were obtained using $N=16$ modes, but using e.g. 160 modes provides no visible change to the graphs. For each of the cases (a)-(d) the effect of nonuniformity is shown for, respectively, a vanishing nonuniformity for reference (zero coefficient, shown with circle symbols for the numerical results), a small nonuniformity corresponding to realistic cases and also to the assumptions underlying the analytical approximations (coefficient 0.1 , squares), and an unrealistically large nonuniformity for which the assumptions underlying the analytical approximation fails substantially (coefficient 1.0, diamonds; e.g. $m_{1}=1.0$ implies an added mass equal to the total pipe mass, i.e. not "small" as assumed). As for the analytical predictions, these predict the phase shift not to depend on nonuniformities in mass or stiffness; consequently the solid lines in each subfigure all overlap, and in particular there is no zero shift. These features are confirmed by numerical simulation, where the marker symbols for zero (circle) or small (square) nonuniformity falls closely on the lines. Only in the cases of unrealistically large nonuniformities (diamonds) do the numerical results visibly differ from the analytical approximations, though not very much so.

Thus, under the assumed smallness in nonuniformity, the equivalence of the simple analytical prediction ((57) with (71)) to the results of detailed numerical solution of a multi-mode expansion is confirmed. Also, the prediction that even a nonuniform mass distribution does not produce a zero shift agrees with experimental findings involving artificially added mass to a real Coriolis flowmeter [20].

\subsubsection{Effect of nonlinear (cubic) damping}

Here we validate the analytical predictions for the case of nonlinear cubic damping, All other imperfections except linear damping and mass flow are ignored, i.e. $\Delta m=\Delta e=\eta=\gamma=L_{k}=\mu$ $=0$, but $\beta \neq 0$ (the coefficient of damping nonlinearity), $\alpha v \neq 0$ (mass flow), and $c_{u}(x)=c_{u 0} \neq 0$ (uniform linear damping, giving $L_{c} \neq 0$ ). 

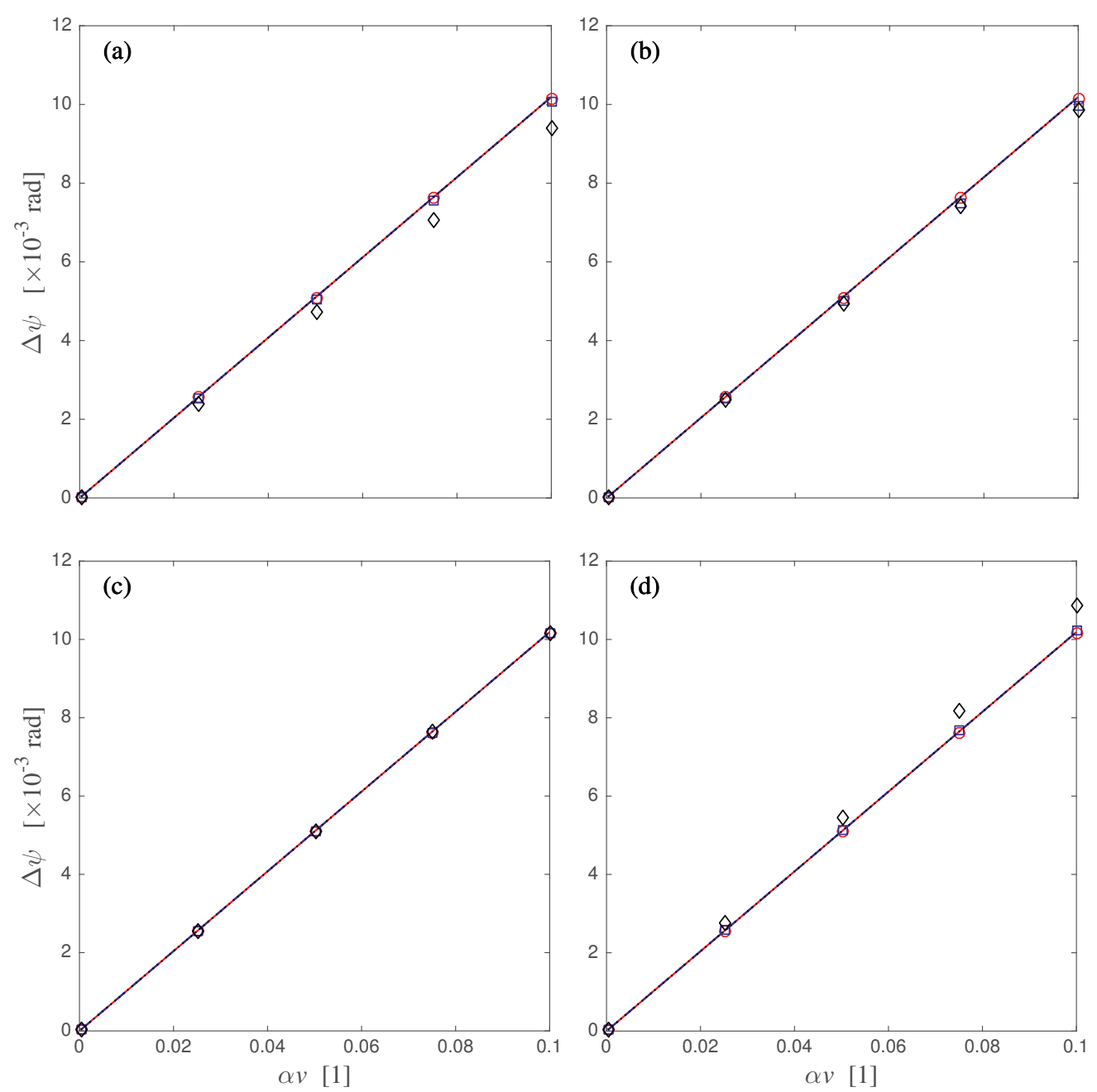

Fig. 4 Phase shift $\Delta \Psi$ for varying mass flow $\alpha N$, with nonuniformity in (a) mass, $m_{1}=\{0,0.1,1\}$; (b) bending stiffness, $e_{1}=\{0,0.1,1\}$; (c) transverse stiffness, $k_{u 1}=\{0,0.1,1\}$; (d) rotational stiffness, $k_{\theta 1}=\{0,0.1,1\}$, as obtained by the analytical approximation (57) with (71) (lines), and by the numerical solution (65)-(66) to (1)(3) using $N=16$ modes ( $\{$ circle, square, diamond $\}$ ) for \{zero, small, large $\}$ nonuniformity). All nonuniformities are localized at $x=0.1$, i.e. $x_{m}=x_{e}=x_{k u}=x_{k} \sigma 0.1$, and damping is taken to be linear and uniformly viscous, i.e. (69) -(70) with $c_{u 0}=0.002$ and $c_{u 1}=c_{\theta 0}=c_{\theta 1}=0$

According to the simple analytical approximation (57) with (71), the phase shift $\Delta \Psi$ does not depend on the coefficient $\beta$ characterizing uniform nonlinear damping; the influence, if any, is predicted to be $O\left(\varepsilon^{3}\right)$. But the stationary vibration amplitude is predicted to be affected. For cubic damping, $f(\dot{u})=\dot{u}^{3}$, we use (45) to calculate the approximate resonant vibration amplitude $\hat{a}^{*}$, using first (32) to calculate $\xi_{1}(x, a)=-\frac{3}{8}\left(\omega_{1} a \varphi_{1}(x)\right)^{3}$, and inserting also (6)-(7) for hinged pipe supports; this gives, for insertion into (45):

$$
\int_{0}^{1} \varphi_{1}(x) \xi_{1}\left(x, \hat{a}^{*}\right) \mathrm{d} x=-\frac{9}{16} \pi^{6}\left(\hat{a}^{*}\right)^{3}, \text { for } f(\dot{u})=\dot{u}^{3} .
$$

When $\beta \neq 0$ Eq. (45) with (73) is a depressed cubic in $\hat{a}^{*}$, which for all relevant physical parameter has only a single real-valued solution [50]):

$$
\hat{a}^{*}=\sqrt[3]{r_{1}+\sqrt{r_{2}}}+\sqrt[3]{r_{1}-\sqrt{r_{2}}}
$$

where 


$$
r_{1}=\frac{4 p \varphi_{1}\left(x_{p}\right)}{9 \beta \pi^{6}}, r_{2}=r_{1}^{2}+\left(\frac{8 c_{11} \omega_{1}}{27 \beta \pi^{6}}\right)^{3} .
$$

For calculating frequency responses in terms of resonant stationary pipe amplitude $\hat{u}\left(x_{1}\right)$ at a sensor location $x_{1}$ as a function of excitation frequency $\Omega$, we use (50) with $\hat{a}$ determined (implicitly, in the nonlinear case) by (42). In (42), by (73) and since $C_{11}=0$ (by (13)) and $\hat{c}_{11}=c_{11}$ (by (13),(15)), the damping term under the radical reduces to:

$$
\hat{c}_{11}-\frac{2 \beta}{\omega_{1} \hat{a}} \int_{0}^{1} \varphi_{1}(x) \xi_{1}(x, \hat{a}) \mathrm{d} x=c_{11}+\frac{9}{8} \pi^{4} \beta \hat{a}^{2}, \text { for } f(\dot{u})=\dot{u}^{3} .
$$

Thus (42) gives, by (43), (6), (7), (20), (12), (11)-(15), and with no stiffness nonlinearity $(\mu=0)$, uniformly distributed mass, stiffness, and cross section $\left(m_{11}=k_{11}=n_{11}=0\right)$ :

$$
\Omega=\omega_{1}-\frac{1}{2} \alpha v^{2} \pm \frac{1}{2} \sqrt{2\left(\frac{p}{\omega_{1} \hat{a}}\right)^{2}-\left(c_{11}+\frac{9}{8} \pi^{4} \beta \hat{a}^{2}\right)^{2}},
$$

which can be solved for $\hat{a}$. Then (50) gives, since $\varphi_{1}\left(x_{1}\right)=\varphi_{1}\left(x_{2}\right)=1$ and $m_{21}=K_{21}=0$ :

$$
\hat{u}\left(x_{1}\right)=\hat{u}\left(x_{2}\right)=\hat{a}+O\left(\varepsilon^{2}\right)
$$

To test the theoretical predictions, Fig. 5(a) first shows the phase shift $\Delta \Psi$ for varying mass flow $\alpha v$ as obtained by the analytical approximation (57) with (71) (lines), and by the numerical solution (65)-(66) to (1)-(3) using $N=8$ modes (symbol markers). Three lines are shown, corresponding to different levels of damping nonlinearity, $\beta=\left\{0,10^{5}, 10^{6}\right\}$; however, since according to (57) $\Delta \Psi$ is independent on $\beta$, the lines are identical. Numerical simulation results (symbol markers) confirms this, agreeing very closely to the analytical prediction.

To ensure this insensitivity to $\beta$ is not just due to parameters corresponding to a very weak damping nonlinearity, the largest value $10^{6}$ used for $\beta$ in Fig. 5(a) was chosen so as to correspond to a damping nonlinearity strong enough to substantially change the frequency response near resonance. This is illustrated by the example frequency response in Fig. 5(b), showing the resonant stationary pipe amplitude $\hat{u}\left(x_{1}\right)$ as a function of excitation frequency $\Omega$ for the largest mass flow $\alpha \nu=0.1$ of Fig. 5(a), and $\beta=10^{6}$, with the theoretical prediction (78) (with $\hat{a}$ from (77)) in solid line. Apparently the nonlinear part of the damping is here strong enough to reduce the peak resonant vibration amplitude to about half the value for linear damping only (dashed line). The circle mark shows the result of numerical simulation ((9) with (59)-(60)), agreeing closely with the theoretical prediction (78) with (74) (max value on solid line).

Next we illustrate the modal composition of the pipe vibrations, i.e. the significance of different modes. In Fig. 6 the top graph shows a simulation time series for the stationary displacement responses $u\left(x_{1}, t\right)$ and $u\left(x_{2}, t\right)$ at sensor measurement locations $x_{1}$ and $x_{2}$, for parameters corresponding to the circle marker in Fig. 5(b). The two responses appear indistinguishable at this scale, the phase shift between them being so small (of order magnitude $10^{-2}$, cf. Fig. 5(a)), corresponding to a time shift $\left.\Delta t=\Delta \Psi / \Omega \approx 10^{-3}\right)$. The lower graphs show the first four modal amplitudes $q_{1-4}(t)$, i.e. the lowest terms in the Galerkin expansion (9) for $u\left(x_{1,2}, t\right)$. Apparently $q_{1}$ alone is indistinguishable from $u\left(x_{1,2, t}\right)$, reflecting the strong dominance of the resonantly excited first mode. The modal amplitude $q_{2}$ of the second mode (main responsible for the phase shift of interest) is two orders of magnitude smaller than $q_{1}$, and the third and fourth modes even smaller (four orders of magnitude); for modes 5-16 the modal amplitudes (not shown) 
are even smaller. This explains why even a greatly reduced 2-mode model, as the one employed in sects. 3-4 for deriving analytical response predictions, provides high accuracy and good agreement with numerical simulation using many modes.

Summing up this section, the analytical prediction that uniformly distributed nonlinear damping does not affect phase shift appears validated by numerical simulation, even for levels of nonlinearity strong enough to significantly affect pipe vibration amplitude. Also, even with a significant level of nonlinearity, analytical predictions based on just the two lowest linear mode shapes shows excellent agreement with numerical simulation using many modes. Numerical simulation was performed only for the case of cubic damping. However, this form of damping can be seen as rather generic, representing the essence of many types of real damping forces that are antisymmetric in relative velocity. This includes "quadratic" or "air" damping $\left(f(\dot{u})=\dot{u}^{2} \operatorname{sgn}(\dot{u})\right)$, and generally any damping law that is oddly symmetric in $\dot{u}$ and can be Taylor-expanded at $\dot{u}=0$ for small $\dot{u}$; the cubic term is then the dominating nonlinearity.

\subsubsection{Effect of nonlinear (cubic) stiffness}

Next we validate the analytical predictions for the case of cubic stiffness nonlinearity, as will be relevant e.g. for applications with constrained axial motion, leading to midplane stretching ( $\mu \neq 0$, cf. definition of $\mu$ in (5) and the discussion in Sect. 2.3, 2nd paragraph). All other imperfections except linear damping and mass flow are ignored, i.e. $\Delta m=\Delta e=\eta=\gamma=L_{k}=\beta=$ 0 , but $\mu \neq 0$ (the coefficient for cubic stiffness nonlinearity), $\alpha v \neq 0$ (mass flow), and $c_{u}(x)=c_{u} 0$ $\neq 0$ (uniform linear damping, giving $L_{c} \neq 0$ ).

As for the case with nonlinear damping, according to the simple analytical approximation (57) with (71), the phase shift $\Delta \Psi$ is predicted not to depend on the nonlinear stiffness coefficient $\mu$, while the stationary vibration amplitudes will be affected.

Using again (45) with (6)-(7) (for hinged pipe supports) to calculate the approximate resonant vibration amplitude $\hat{a}^{*}$, one finds:
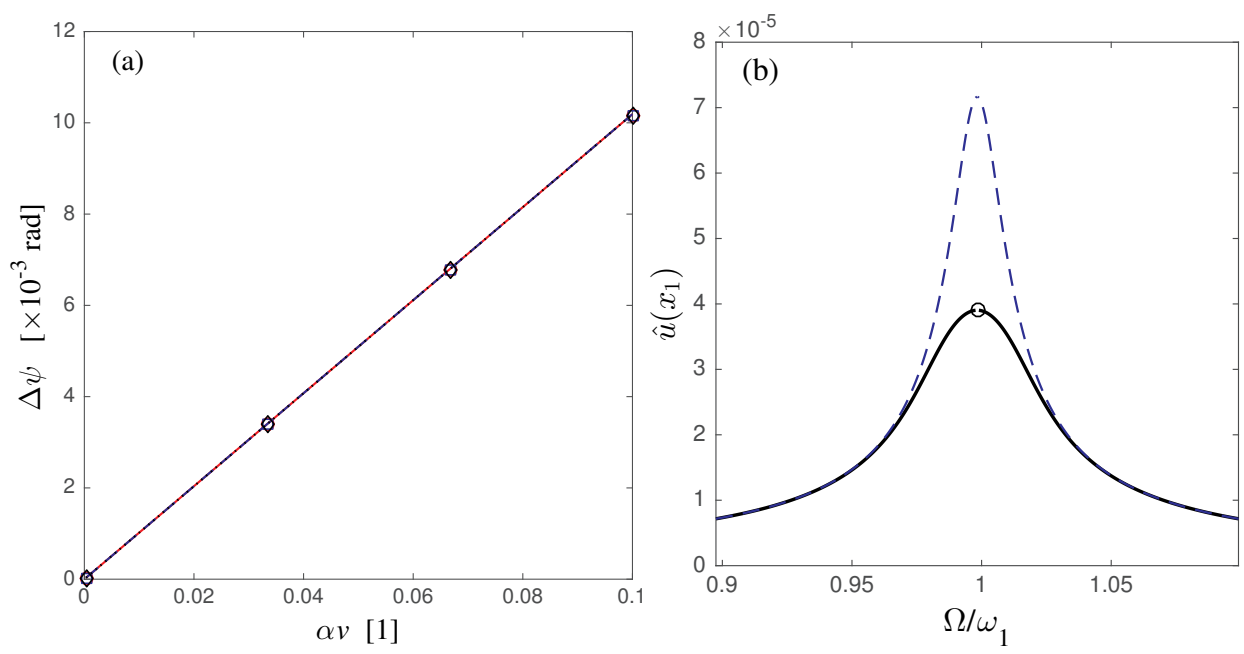

Fig. 5 (a) Effect of nonlinear (cubic) damping $f(\dot{u})=\dot{u}^{3}$ on phase shift $\Delta \Psi$ for varying mass flow $\alpha v$, as obtained by the analytical approximation (57) with (71) (lines), and by the numerical solution (65)-(66) to (1)-(3) using $N=8$ modes (symbol markers). The lines show $\Delta \Psi$ for different levels of damping nonlinearity $\beta=\left\{0,10^{5}, 10^{6}\right\}$ with $c_{u 0}=0.2$ and $p=10^{-4}$ in all cases. (b) Frequency response showing the resonant stationary pipe amplitude $\hat{u}\left(x_{1}\right)$ as a function of excitation frequency $\Omega$ when $\alpha v=0.1$. Solid line: analytical prediction (78) (with $\hat{a}$ from (77)) for cubic damping $\left(\beta=10^{6}\right)$; dashed line: linear damping $(\beta=0)$; circle marker: numerical simulation $((9)$ with (59)-(60)) 


$$
\hat{a}^{*}=\frac{\sqrt{2}}{\pi^{2}} \frac{p}{c_{11}} .
$$

That is, by contrast to the above case of nonlinear cubic damping, nonlinear cubic stiffness does not affect the peak response amplitude. It only affects the frequency of excitation where the peak amplitude is encountered, i.e. it changes the backbone of the frequency response curve.

For calculating frequency responses we use the same procedure as described in Sect. 5.2.4 for nonlinear damping, i.e. $\hat{u}\left(x_{1}\right)$ is computed from (50) with $\hat{a}$ determined by (42). This gives, instead of (77):

$$
\Omega=\omega_{1}-\frac{1}{2} \alpha v^{2}+\frac{3}{16} \mu^{2} \omega_{1} \hat{a}^{2} \pm \frac{1}{2} \sqrt{2\left(\frac{p}{\omega_{1} \hat{a}}\right)^{2}-c_{11}{ }^{2}},
$$

which can be solved for $\hat{a}$. Then $\hat{u}\left(x_{1}\right)$ is given by (50), again with $m_{21}=K_{21}=0$ :

$$
\hat{u}\left(x_{1}\right)=\hat{u}\left(x_{2}\right)=\hat{a}+O\left(\varepsilon^{2}\right)
$$
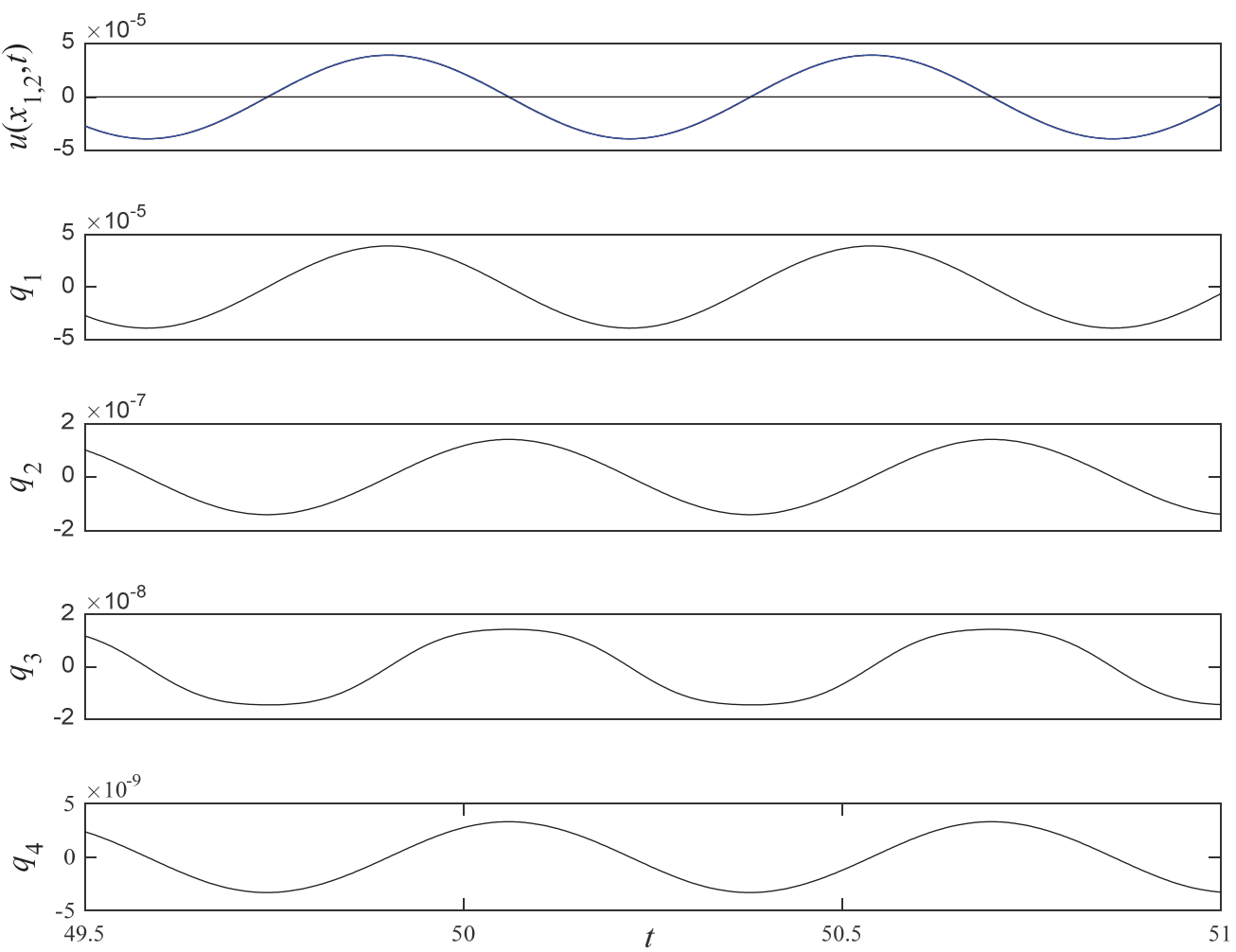

Fig. 6 Stationary resonant response for a case of nonlinear damping, with system parameters corresponding to the circle marker in Fig. 5(b). Top: Displacement response $u\left(x_{1}, t\right)$ and $u\left(x_{2}, t\right)$ (difference too small to be visible at this scale) at measurement locations $x_{1}$ and $x_{2}$ computed by (9) with $N=8$ modes; lower graphs: Modal amplitudes $q_{j}(t)$ for the four lowest modes 
To test the theoretical predictions, Fig. 7(a) shows the phase shift versus mass flow as obtained by the analytical approximation (57) with (71) (lines), and by the numerical solution (65)-(66) to (1)-(3) using $N=8$ modes (symbol markers). Three lines are shown, corresponding to different levels of stiffness nonlinearity, $\mu=\{0,3000,7000\}$; however, since according to (57) $\Delta \Psi$ is independent on $\mu$, the lines are identical (and also identical to the lines in Fig. 5(a)). Numerical simulation results (symbol markers) agree closely with the theoretical predictions, except for the largest value of $\mu=7000$ where a small deviation is visible. However, this value of $\mu$ is unrealistically large, and chosen just to provoke at least some visibly detectable change in phase shift. For the particular (rather typical) industrial flowmeter, whose parameter values provide numerical illustration for this work, the slenderness ratio $\mu$ is about 300 . This means the highest value for $\mu$ used with Fig. 7 is more than twenty times that of the example flowmeter, and even the "medium" value of $\mu=3000$, which still gives excellent agreement between analytical results and numerical simulation, is one order of magnitude larger than for the typical flowmeter. Thus, for a real flowmeter, one can expect uniformly distributed cubic nonlinearity (as from e.g. midplane stretching) to have insignificant effect on phase shift. A similar observation was reported in [28] for a mathematical model of a curved micro Coriolis flowmeter, employing Galerkin-based numerical solutions: For the specific numerical example reported, the mass flow remains linearly related to phase shift, even in the presence of cubic stiffness nonlinearity.

As for the case with nonlinear damping, though the phase shift is not affected by uniformly distributed nonlinearity, the frequency response $i s$ affected. To illustrate this for the case of nonlinear stiffness, Fig. 7(b) shows the resonant stationary pipe amplitude $\hat{u}\left(x_{1}\right)$ as a function of excitation frequency $\Omega$ for the largest mass flow $\alpha v=0.1$ of Fig. 7(a), and $\mu=7000$; The theoretical prediction (81) (with $\hat{a}$ from (80)) is shown in solid line, with the corresponding linear response $(\mu=0)$ dashed. With such a large value of $\mu$ the resonant response peak is significantly bent towards higher frequencies, a well-known feature with hardening nonlinearity $(\mu>0$; e.g. [26]). The circle marker shows the result of numerical simulation ((9) with
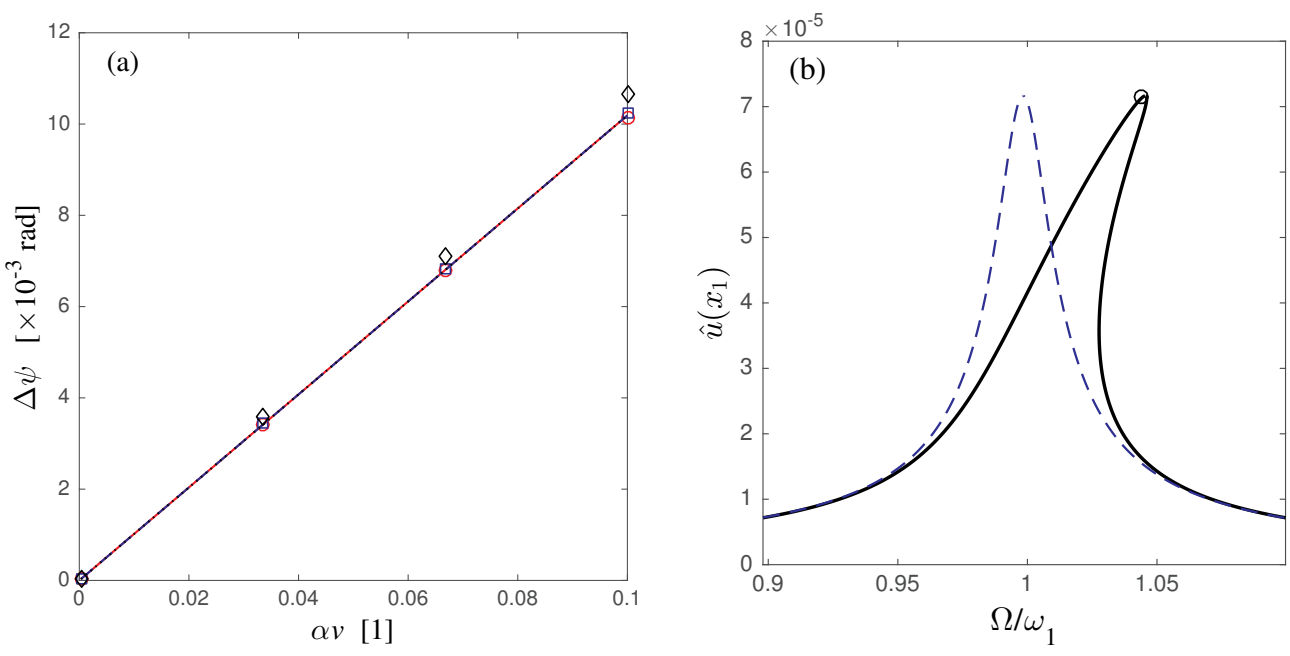

Fig. 7 (a) Effect of nonlinear (cubic) stiffness on phase shift $\Delta \Psi$ for varying mass flow $\alpha v$, as obtained by the analytical approximation (57) with (71) (lines), and by the numerical solution (65)-(66) to (1)-(3) using $N=8$ modes (symbol markers). The lines show $\Delta \Psi$ for different levels of stiffness nonlinearity $\mu=\{0,3000,7000\}$ with $c_{u 0}=0.2$ and $p=10^{-4}$ in all cases. (b) Example frequency response showing the resonant stationary pipe amplitude $\hat{u}\left(x_{1}\right)$ as a function of excitation frequency $\Omega$ when $\alpha v=0.1$. Solid line: analytical prediction (81) with (80) for cubic stiffness $(\mu=7000)$; dashed line: linear stiffness $(\mu=0)$; circle marker: numerical simulation ( $(9)$ with (59)-(60)) 
(59)-(60)), agreeing rather closely with the theoretical prediction (79) (max value on solid line); for lower (more realistic values of $\mu$ ) the peak is less bent, and the agreement with numerical simulation even closer.

As for the contribution of various modes to the nonlinear resonant response, graphs of $u\left(x_{1,2, t}\right)$ and the modal amplitudes $q_{j}(t)$ show features very similar as for the case of nonlinear damping (cf. Fig. 6); they are thus not shown here. Again the response is strongly dominated by the first mode, with the modal amplitude of the second mode being two orders of magnitude smaller, and that of the third and higher modes four or more orders of magnitudes smaller. Again this contributes to explaining why the reduced 2-mode model provides good agreement to numerical simulation using many modes.

\section{Conclusions}

A mathematical model of a resonantly excited vibrating pipe with fluid flow was set up, including generic models of several imperfections relevant for applications, in particular in Coriolis flowmetering: Small axial variations (possibly discontinuous) in pipe mass, bending stiffness, and transverse and rotational linear viscous damping and stiffness (additional to pipe bending stiffness), axial pre-tension, nonlinear stiffness (quadratic-cubic), and generalized velocity-dependent nonlinear damping. The imperfections serve as simple theoretical models for many features of real flowmeters, such as mounted sensors and actuators, production inaccuracies, mounting conditions (e.g. constrained pipe ends), and wear, contamination, and corrosion.

Assuming imperfections to be small, a two-mode approximation was employed, and perturbation analysis used to calculate approximate analytical predictions for the frequency response, and for the vibration phase shift between two points along the pipe; the latter is the prime measured quantity in Coriolis flowmetering. The analytical expression (Eq. (52)) for the phase shift is very simple, though accurate to second order; it allows direct insight into how parameters other than mass flow affects phase shift, and thus in Coriolis flowmetering could be mistaken for mass flow. The following readily appear from inspecting the analytical prediction for phase shift:

The meter sensitivity (ratio of phase shift to mass flow) is predicted to a) grow with the nearness of the natural frequencies of two lowest vibration modes, in the same proportion as reported in other studies $[1,45]$; b) be independent on the vibration amplitude, and c) be independent on all imperfections included in this study, i.e. independent of small linear and nonlinear damping, mass and stiffness nonuniformity, and additional transverse stiffness (by pretension, midplane stretching / symmetric stiffness nonlinearity, or asymmetric nonlinearity). Also, a zero phase shift (nonzero shift even in absence of mass flow) may result if the damping distribution is not symmetric along the pipe axis, but not with any of the other imperfections considered.

The main assumption for calculating the simple analytical approximation for the phase shift is that the imperfections considered are small, and that the vibration modes of importance is the first (which is resonantly excited) and the second (which is weakly excited by Coriolis forces associated with mass flow and certain imperfections), while the influence of all higher modes is negligible in comparison. It then follows from the analysis that if the imperfections considered are of magnitude order $O(\varepsilon), \varepsilon \ll 1$, then the effect of asymmetrically distributed damping on phase shift is of the same order of magnitude as the mass flow, i.e. also $O(\varepsilon)$, and introduces a zero shift that could be mistaken for mass flow, while the effect of all other imperfections considered are at least two orders of magnitude, smaller, i.e. $O\left(\varepsilon^{3}\right)$. 
Validation of the analytical expression was performed by comparing to results of numerical simulation of the full equation of motion, using a Galerkin expansion with many modes, and for parameter sets for various imperfections ranging from "small" (as assumed, realistically) to "large" (orders of magnitude larger than realistic). In all cases of "small" imperfections agreement were excellent; only with "large" imperfections deviations show up.

The numerically based validation was performed for a broad range of parameters for the imperfections considered. But naturally it is not possible to test every imperfection considered thoroughly in a full parameter space; for example, an endless variation of possible relevant spatial distributions of even linear damping exists, and the same for "nonlinear damping". However, for imperfections that could be of practical interest for applications, the simple analytical approximations for the phase shift (Eq. (52)) and the stationary vibration amplitude (Eq. (50)) can be used to rather easily predict whether or not a given imperfections affects phase shift and how, possibly cross-checking with numerical simulation or laboratory experiments.

The theoretical prediction that asymmetrically distributed damping affects phase shift, while a symmetric damping distribution or small nonuniformity in mass distribution has no significant effect, agrees with earlier reported experimental findings [20,49]. Also, the theoretical prediction that weak cubic stiffness type nonlinearity does not affect phase shift agrees with recently reported numerical observations for a particular curved micro Coriolis flowmeter [28]. However, the remaining of the abovementioned predictions of effects of various imperfections should be considered theoretically based hypotheses, awaiting experimental validation.

\section{Compliance with Ethical Standards}

Conflict of interest: The second author received financial support for traveling and accommodation from Siemens Flow Instruments in Denmark.

\section{References}

[1] Thomsen, J.J, Dahl, J.: Analytical predictions for vibration phase shifts along fluid-conveying pipes due to Coriolis forces and imperfections. Journal of Sound and Vibration 329, 3065-3081 (2010)

[2] Benjamin, T.: Dynamics of a system of articulated pipes conveying fluid - I. Theory. Proceedings of the Royal Society A 261, 457-486 (1961)

[3] Benjamin, T.: Dynamics of a system of articulated pipes conveying fluid - II. Experiments. Proceedings of the Royal Society A 261, 487-499 (1961)

[4] Païdoussis, M.P, Issid, N.T.: Dynamic stability of pipes conveying fluid. Journal of Sound and Vibration 33, 267-294 (1974)

[5] Païdoussis, M.P.: Fluid Structure Interactions: Slender Structures and Axial Flow, Vol. 1. Academic Press, London (1998)

[6] Païdoussis, M.P.: The canonical problem of the fluid-conveying pipe and radiation of the knowledge gained to other dynamics problems across Applied Mechanics. Journal of Sound and Vibration 310, 462-492 (2008)

[7] Ibrahim, R.A.: Mechanics of Pipes Conveying Fluids - Part I: Fundamental Studies. Journal of Pressure Vessel Technology 132, 034001-1:32 (2010)

[8] Ibrahim, R.A.: Mechanics of Pipes Conveying Fluids - Part II: Applications and Fluidelastic Problems. Journal of Pressure Vessel Technology 133, 024001-1:30 (2011)

[9] Anklin, M., Drahm, W., Rieder, A.: Coriolis mass flowmeters: overview of the current state of the art and latest research. Flow Measurement and Instrumentation 17, 317-323 (2006)

[10] Ghayesh, M.H., Amabili, M., Païdoussis, M.P.: Thermo-mechanical phase-shift determination in Coriolis mass-flowmeters with added masses. Journal of Fluids and Structures 34, 1-13 (2012) 
[11] Wang, T, Baker, R.: Coriolis flowmeters: a review of developments over the past 20 years, and an assessment of the state of the art and likely future directions. Flow Measurement and Instrumentation 40, 99-123 (2014)

[12] Raszillier, H, Durst, F.: Coriolis-effect in mass flow metering. Archive of Applied Mechanics 61, 192-214 (1991)

[13] Lange, U., Levient, A., Pankratz, T., Raszillier, H.: Effect of detector masses on calibration of Coriolis flowmeters. Flow Measurement and Instrumentation 5, 255-262 (1994)

[14] Sultan, G, Hemp, J.: Modelling of the Coriolis mass flowmeter. Journal of Sound and Vibration 132, 473 489 (1989)

[15] van de Ridder, L., Hakvoort, W., van Dijk, J., Lötters, J., de Boer, A.: Quantification of the influence of external vibrations on the measurement error of a Coriolis mass-flowmeter. Flow Measurement and Instrumentation 40, 39-49 (2014)

[16] Cheesewright, R, Clark, C.: The effect of flow pulsations on Coriolis mass flow meters. Journal of Fluids and Structures 12, 1025-1039 (1998)

[17] Belhadj, A., Cheesewright, R., Clark, C.: The simulation of Coriolis meter response to pulsating flow using a general purpose F.E. code. Journal of Fluids and Structures 14, 613-634 (2000)

[18] Enz, S, Thomsen, J.J.: Predicting phase shift effects for vibrating fluid-conveying pipes due to Coriolis forces and fluid pulsation. Journal of Sound and Vibration 330, 5096-5113 (2011)

[19] Svete, A., Kutin, J., Bobovnik, G., Bajsic, I.: Theoretical and experimental investigations of flow pulsation effects in Coriolis mass flowmeters. Journal of Sound and Vibration 352, 30-45 (2015)

[20] Enz, S., Thomsen, J.J., Neumeyer, S.: Experimental investigation of zero phase shift effects for Coriolis flowmeters due to pipe imperfections. Flow Measurement and Instrumentation 22, 1-9 (2011)

[21] Basse, N.T.: Coriolis flowmeter damping for two-phase flow due to decoupling. Flow Measurement and Instrumentation 26, 40-52 (2016)

[22] Kutin, J, Bajsic, I.: Stability-boundary effect in Coriolis meters. Flow Measurement and Instrumentation 12 , 65-73 (2001)

[23] Kutin, J, Bajsic, I.: An analytical estimation of the Coriolis meter's characteristics based on modal superposition. Flow Measurement and Instrumentation 12, 345-351 (2002)

[24] Nayfeh, A.H, Mook, D.T.: Nonlinear Oscillations. Wiley, New York (1979)

[25] Nayfeh, A.H.: Nonlinear Interactions. Wiley, New York (1997)

[26] Thomsen, J.J.: Vibrations and Stability: Advanced Theory, Analysis, and Tools. Springer-Verlag, Berlin Heidelberg (2003)

[27] Dezhi, Z., Shuai, W., Shangchun, F.: Nonlinear vibration characteristics of Coriolis mass flowmeter. Chinese Journal of Aeronautics 22, 198-205 (2009)

[28] Ghazavi, M., Molki, H., Ali Beigloo, A.: Nonlinear vibration and stability analysis of the curved microtube conveying fluid as a model of the micro coriolis flowmeters based on strain gradient theory. Applied Mathematical Modelling 45, 1020-1030 (2017)

[29] Enz, S.: Effect of asymmetric actuator and detector position on Coriolis flowmeter and measured phase shift. Flow Measurement and Instrumentation 21, $497-503$ (2010)

[30] Wang, T., Baker, R.C., Hussain, Y.: An advanced numerical model for single straight tube Coriolis flowmeters. Journal of Fluids Engineering 128, 1346-1350 (2006)

[31] Wang, S., Clark, C., Cheesewright, R.: Virtual Coriolis flow meter: a tool for simulation and design. Proceedings of the Institution of Mechanical Engineers, Part C: Journal of Mechanical Engineering Science 220, 817-835 (2006)

[32] Mole, N., Bobovnik, G., Kutin, J., Stok, B., Bajsic, I.: An improved three-dimensional coupled fluid-structure model for Coriolis flowmeters. Journal of Fluids and Structures 24, 559-575 (2008)

[33] Ruoff, J., Hodapp, M., Kück, H.: Finite element modelling of Coriolis mass flowmeters with arbitrary pipe geometry and unsteady flow conditions. Flow Measurement and Instrumentation 37, 119-126 (2014) 
[34] Bobovnik, G., Kutin, J., Mole, N., Štok, B., Bajsic, I.: Numerical analysis of installation effects in Coriolis flowmeters: Single and twin tube configurations. Flow Measurement and Instrumentation 44, 71-78 (2015)

[35] Binulal, B., Rajan, A., Kochupillai, J.: Dynamic analysis of Coriolis flowmeter using Timoshenko beam element. Flow Measurement and Instrumentation 47, 100-109 (2016)

[36] Yaushev, A.A., Taranenko, P.A., Loginovskiy, V.A.: Analysis of influence of mechanical boundary conditions on zero point shift of Coriolis flowmeter. Procedia Engineering 206, 552-557 (2017)

[37] Cheesewright, R., Clark, C., Belhadj, A., Hou, Y.Y.: The dynamic response of Coriolis mass flow meters. Journal of Fluids and Structures 18, 165-178 (2003)

[38] Kutin, J., Bobovnik, G., Hemp, J., Bajsic, I.: Velocity profile effects in Coriolis mass flowmeters: recent findings and open questions. Flow Measurement and Instrumentation 349-358 (2006)

[39] Hemp, J, Kutin, J.: Theory of errors in Coriolis flowmeter readings due to compressibility of the fluid being metered. Flow Measurement and Instrumentation 17, 359-369 (2006)

[40] Lautrup, B.: Physics of Continuous Matter: Exotic and Everyday Phenomena in the Macroscopic World. Institute of Physics, Bristol, Philadelphia (2005)

[41] Shabana, A.A.: Vibration of Discrete and Continuous Systems (2nd Ed.). Springer-Verlag, New York (1997)

[42] Ziegler, H.: Principles of Structural Stability. Blaisdell, Waltham, Massachusetts (1968)

[43] Nayfeh, A.H.: Perturbation Methods. Wiley, New York (1973)

[44] Kevorkian, J, Cole, J.D.: Multiple Scale and Singular Perturbation Methods. Springer-Verlag, New York (1996)

[45] Baker, R.C.: Flow Measurement Handbook. Cambridge University Press, Cambridge (2000)

[46] Raszillier, H., Alleborn, N., Durst, F.: Effect of a concentrated mass on coriolis flowmetering. Archive of Applied Mechanics 64, 373-382 (1994)

[47] Ewins, D.J.: Modal Testing: Theory, Practice and Application (2nd Ed.). Research Studies Press Ltd., Hertfordshire, England (2000)

[48] Cunningham, T.J.: Zero shifts due to non-proportional damping. In:, Proceedings of the 15th International Modal Analysis Conference, IMAC, Orlando, Florida, Feb. 3-6, 1997, Vol. 1, pp. 237-243 (1997)

[49] Thomsen, J.J., Dahl, J., Fuglede, N., Enz, S.: Predicting phase shift of elastic waves in pipes due to fluid flow and imperfections. In: M.R. Pawelczyk, D. Bismor (Eds.), Proceedings of the 16th International Congress on Sound and Vibration (ICSV16), Kraków, Poland, July 5-9, 2009, 8pp. (2009)

[50] Mathews, J.H, Howell, R.W.: Complex Analysis for Mathematics and Engineering (4th Ed.). Jones \& Bartlett, Sudbury, MA, USA (2001) 